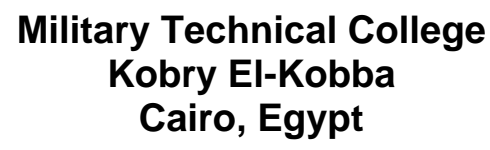

12-th International Conference

on

Aerospace Sciences \&

Aviation Technology

\title{
EVOLUTION OF HEAVY PARTICLE TRIANGULAR CLUSTERS IN 3-D TURBULENT FLOW
}

\author{
A. ELMaihy ${ }^{*}$ A. Abou EL-Azm Aly**, F. Nicolleau ${ }^{* * *}$
}

\begin{abstract}
The dispersion of heavy particle triangular clusters in a homogenous, isotropic and incompressible 3-D turbulent flow have been studied using the Kinematic Simulation techniques. In this study, the evolution of the size and the shape factors of the cluster are presented at different initial separations between particles, different inertia effect (different Stokes numbers), and different values of drift velocities.

For dispersion of heavy particles in turbulent flows It can be concluded that the inertia and gravity affect the size and shape of the three-particles cluster. For the effect of Reynolds number on the dispersion of heavy particle clusters, Nicolleau and EIMaihy (2006) found that all the curves for constant values of initial separations of $\Delta_{0} / L_{1}=$ 0.032 and $\Delta_{0} / L_{1}=0.5$ are approximately collapsed for factors $\left\langle R^{2}\right\rangle^{1 / 2},\langle W\rangle$ and $\langle\chi\rangle$ ]. These results can be extended to the heavy particle dispersion for Stokes numbers in the range $0.2<\mathrm{St}<1$ at zero drift velocity for inertial ranges; $185<\mathrm{k}_{\mathrm{N}} / \mathrm{k}_{1}<2000$. However, in the present work a limitation has been introduced for the above conclusion to initial separations above the Kolmogorov length-scale.
\end{abstract}

\section{KEYWORDS}

Heavy particles, Kinematic Simulation, particle cluster, two phase flow.

\footnotetext{
* Egyptian Armed Forces

** PhD Student. Department of Mechanical Engineering, University of Sheffield, Sheffield, UK

*** Senior Lecturer: electronic address; F.Nicolleau@Sheffield.ac.uk
} 


\section{NOMENCLUTRE}

\begin{tabular}{|c|c|c|c|}
\hline a & particle radius & $\mathrm{dt}$ & time increment \\
\hline \multirow[t]{2}{*}{$A_{n}, B_{n}$} & amplitudes corresponding to the & $E(k)$ & Eulerian energy spectrum \\
\hline & vectors $\overrightarrow{\mathbf{A}}_{n}$ and $\overrightarrow{\mathbf{B}}_{n}$ & $\mathrm{R}$ & Radius of gyration \\
\hline d & particle diameter & $g$ & acceleration of gravity \\
\hline \multirow[t]{2}{*}{$\mathrm{du} / \mathrm{dt}$} & time derivative following the moving & $t_{n}$ & Kolmogorov microscale time \\
\hline & particle & $\mathrm{R}(\mathrm{t}, \mathrm{T})$ & Correlation function \\
\hline \multirow[t]{3}{*}{$\mathrm{Du} / \mathrm{Dt}$} & time derivative of the fluid velocity & $\operatorname{Re}_{\mathrm{p}}$ & particle Reynolds number \\
\hline & & W & $=\rho_{\mathrm{f}} \mathrm{vd} / \mu$ \\
\hline & & & drift velocity parameter \\
\hline k & Wavenumber & G & $\mathrm{i}^{\text {th }}$ eigen value of the moment of \\
\hline $\mathrm{k}_{\mathrm{N}}$ and $\mathrm{k}_{\mathrm{n}}$ & highest wavenumber & & inertia like tensor \\
\hline $\mathrm{k}_{1}$ and $\mathrm{k}_{\mathrm{L} 1}$ & lowest wavenumber & $u_{i}^{\prime}$ & turbulent intensity \\
\hline $\mathrm{L}$ & characteristics length scale & $\mathrm{I}_{2}$ & shape geometry parameter \\
\hline $\mathrm{L}_{1}$ & Largest length scale & $\overrightarrow{\mathbf{K}}_{n}$ & $\mathrm{n}^{\text {th }}$ wave vector \\
\hline$m_{p}$ & mass of spherical particle & $\mathrm{kn}$ & magnitude of wavevector \\
\hline$m_{f}$ & equivalent mass of displaced fluid & $\hat{\mathbf{K}}_{n}$ & Unit vector of wavevector \\
\hline$N_{k}$ & number of modes & $\operatorname{Re}$ & Reynolds number \\
\hline$t_{d}$ & Eddy turn over time scale & $\mathrm{R}_{\lambda}$ & Reynolds number based on \\
\hline St & Stokes umber $=\mathrm{T}_{\mathrm{a}} / \mathrm{t}_{\mathrm{d}}$ & & Taylor length micro-scale \\
\hline $\mathrm{u}(\mathrm{x}, \mathrm{t})$ & Eulerian velocity field & w & Shape geometry parameter \\
\hline$v\left(x_{p}, t\right)$ & velocity of the particle & $x$ & fluid displacement \\
\hline & particle position $x_{p}$ & $\mathrm{~T}_{\mathrm{a}}$ & particle dynamic response time \\
\hline$x_{p}$ & Particle displacement & $\lambda$ & Unsteadiness coefficient \\
\hline$u\left(x_{p}, t\right)$ & $\begin{array}{l}\text { velocity of the fluid at the particle } \\
\text { position }\end{array}$ & $\omega_{\mathrm{n}}$ & $\begin{array}{l}\text { Unsteadiness frequency } \\
\text { Fourier mode } \mathrm{n} \text {. }\end{array}$ \\
\hline$V_{d}$ & terminal fall velocity in still fluid & $\eta$ & Kolmogorov length scale. \\
\hline$<>$ & ensemble averaging over many & $x$ & Shape geometry parameter \\
\hline & realization. & $\Delta_{0}$ & Initial separation \\
\hline
\end{tabular}

\section{INTRODUCTION:}

One of the major problems existing in turbulence is the existence of two phase flow such as water bubbles in air or gaseous bubbles in water or vapour bubbles in oil as in the fluid power systems carrying turbulent flow. Understanding the turbulent flow 
features, especially these of the two phase regime in which the dispersion of heavy particle clusters plays a significant role in the three-dimensional turbulent flow, and as a consequence the factors affecting this dispersion process, may help in the analysis of such systems and may also shed some light on the turbulent flow details in two phase flow and give information about the turbulence fluctuations.

Because of the recent developments in methods for tracking particles, one can now easily follow more than one or two particles making it possible to study not only the statistics of the relative dispersion of particles and their separation evolution, but also their geometry evolution.

The term "Particle dispersion" is used to define the dispersion of particles with inertia and other effects due to the fact that they are different from the carrier fluid as their densities are larger than the fluid. Industrial applications of heavy particle turbulent dispersion are concerned with the design and control of various multiphase processes such as droplets formation for combustion in furnaces, diesel engines or droplets of liquids in cyclone separation and pneumatic systems.

The dispersion process of heavy particle clusters is characterized by both the properties of the heavy particles and the properties of the turbulent fluid that carries the particles such as the effect of the buoyancy force acting on the particle and the effect of the relative motion between the particle and the turbulent flow field. These effects cause the velocity of the particle to be different from that of the surrounding fluid. Hence, the trajectories between the heavy particle and that of the fluid element in the flow are also different. Consequently, tracking of heavy particles is more difficult than the diffusion of small light particles.

The importance of studying the evolution of $n$-particles in turbulent flow was addressed by Mydlarski et al [1] to study the n-order moments (higher order structure function) which was considered as a way to connect the scaling properties of turbulence to the spatial structure of the flow. Particle inertia produces a bias in each trajectory towards regions of high strain rate (convergence zones) and low vorticity which affects the mean settling velocity. Pumir et al [2] investigated the statistical geometry of Lagrangian trajectories of $n=3,4$ clusters of material particles in threedimensional turbulent flows either by using Direct Numerical Simulations (DNS) at a moderate Reynolds number $\left(R_{\lambda}=82\right)$ that corresponds to inertial sub-range of $\mathrm{k}_{N} / \mathrm{k}_{1}=185$ or by using a simple phenomenological model of Lagrangian kinematics owing to the Reynolds number limitations of DNS. Castiglione and Pumir [3] studied the geometrical aspects of Lagrangian dispersion and the shape distortion of small triangles in an experimental 2-D turbulent flow in the inverse energy cascade regime with a $\mathrm{k}^{-5 / 3}$ spectrum. The experiment results provided strong evidence that the shape of triangles distortion is nontrivial and depends on its initial size. The Lagrangian dispersion of three particles with the help of the kinematic simulation was studied by Khan et al [4] in a two-dimensional turbulent flow. They have used a 2-D flow to make direct comparison with the results obtained previously and investigated the shape distribution of triangles advected in turbulence with a high Reynolds number. Nicolleau and EIMaihy [5] extended the studies done by Khan et al [4] using the kinematic simulation technique to study the effect of the Reynolds number, the initial separation and the unsteadiness term modelling for triangles (three particles) and tetrahedron (four particles) advected in a 3-D turbulent flow.

Here, a homogenous and isotropic incompressible turbulence is the studied domain. In this case, the dispersion of heavy particle triangle clusters in the turbulent flow and 
the evolution of their size and shape factors have been studied at different cases of initial separation between particles, inertia effect and gravity parameters.

The numerical approach used to generate turbulent flow field is introduced in section 2 , the heavy particle equation of motion is presented in section 3 with the considered assumptions. In section 4 , the heavy particle cluster parameterization is introduced. The obtained results for dispersion of heavy particle triangle clusters are discussed in section 5 and the conclusion is presented in section 6.

\section{KINEMATIC SIMULATION MODEL:}

Fung et al [6] extended the work of Kraichnan [7] and Drummond et al [8] and developed a new Lagrangian model of turbulent flow, and called it Kinematic Simulation. Kinematic simulation, KS, is not an Eulerian turbulence model but is based on a kinematical simulation of the Eulerian velocity field. It is generated as a sum of random incompressible Fourier modes whose statistics agree with the values obtained from experimental measurements or other reliable numerical simulations (as opposed to simulation techniques based on the dynamical equations). The flow structures in KS are turbulent-like in the sense that they are of the type of eddying, straining, and streaming structures similar to those expected and observed in real turbulent flows but with no sweeping effect of large scale to small scale eddies. With this method, the computational task in is to calculate the trajectory of each particle placed in the turbulent field. Each trajectory is associated with the differential equation:

$$
\mathrm{u}_{\mathrm{E}}=\mathrm{dx} / \mathrm{dt}
$$

where $u_{E}$ is the Eulerian velocity field and supposed to be known throughout the KS. Trajectories' calculation are independent of each other and calculated using the $4^{\text {th }}$ order predictor corrector method (Adams-Bashforth-Moulton) in which Runge-Kutta 4 is used to compute the first three points in Adams-Bashforth's method.

By knowing the initial position at initial time, the particle position could be calculated using equation (1) at each time step. This kind of computational program does not require the storage of a lot of data with very big tables as in calculation with direct numerical simulation and this is one of the KS advantages.

The KS model could be considered a powerful tool to study the problem of the dispersion of heavy particle clusters in turbulence. It should also be noted that the $\mathrm{KS}$ model, beside its simplicity, is a unified Lagrangian model of one-two- and indeed multi-particle turbulent diffusion where the effects of small scale flow structures on Lagrangian statistics are taken into account (Fung et al. [6], Fung and Vassilicos [9], Malik and Vassilicos [10]).

\subsection{Kinematic Simulation Model Construction:}

The most important target required for studying the turbulence using a Lagrangian approach as KS is to decompose the fluctuations of turbulence into sinusoidal components and study the distribution of turbulent energy among the different turbulent scales is. As in Flohr and Vassilicos [11], the 3-D KS turbulent velocity field in this study is represented by a truncated Fourier series, sum of $\mathrm{N}$ random Fourier modes:

$$
u(x, t)=\sum_{n=1}^{N_{k}}\left[\left(a_{n} \times \hat{k}_{n}\right) \cos \left(k_{n} \cdot x+\omega_{n} t\right)+\left(b_{n} \times \hat{k}_{n}\right) \sin \left(k_{n} \cdot x+\omega_{n} t\right)\right]
$$

where 
Proceeding of the 12-th ASAT Conference, 29-31 May 2007

$\mathrm{N}_{\mathrm{k}} \quad$ number of Fourier modes.

$\hat{k}_{n} \quad$ a random unit vector distributed independently

and uniformly over a unit sphere $\overrightarrow{\mathrm{K}}_{n}=K_{n} \hat{\mathrm{K}}_{n}$

$\vec{a}_{n}$ and $\vec{b}_{n} \quad$ vectors are chosen randomly under certain

constrain that they are normal to $\hat{k}_{n}$.

$\hat{k}_{n}$ is defined as $\hat{k}_{n}=\vec{k}_{n} /\left|k_{n}\right|$ such that a random unit vector is created with its direction randomly distributed in space. To ensure that the velocity field is incompressible $(\nabla . \mathrm{u}=0)$, the Fourier coefficients are written as $\left(a_{n} \times \hat{k}_{n}\right)$ and $\left(b_{n} \times \hat{k}_{n}\right)$ where $a_{n}$ and $b_{n}$ are real random vectors uniformly distributed over all directions and statically independent.

$$
k_{n}=\mid k_{n}\left(\begin{array}{c}
\sin \theta_{n} \cos \phi_{n} \\
\sin \theta_{n} \sin \phi_{n} \\
\cos \theta_{n}
\end{array}\right)
$$

where $\theta_{\mathrm{n}} \in[0, \pi]$ and $\phi_{\mathrm{n}} \in[0,2 \pi]$ are picked randomly in each mode and realization so that the random choice of directions for the $n^{\text {th }}$ wavemode is independent of the random choice of directions for all other modes.

\subsection{Wavenumber distribution:}

The value of $k_{n}$ has to be chosen by discretizing the wavenumber space into a finite number of modes $\mathrm{N}_{\mathrm{k}}$. This descritization of modes could be chosen according to one of the following distributions:

$$
k_{n}= \begin{cases}k_{1}\left(\frac{k_{\eta}}{k_{1}}\right)^{n-1 / N_{k}-1} & \text { geometric disribution } \\ \mathrm{k}_{1} n^{\ln \left(k_{\eta} / k_{1}\right) / \ln N_{k}} & \text { algebraic distribution } \\ k_{1}+\left(\frac{k_{\eta}-k_{1}}{N_{k}-1}\right)(n-1) & \text { linear distribution }\end{cases}
$$

with $n \in\left[1, N_{k}\right]$

Here, the geometric distribution has been chosen because it leads to equally spaced energy shells for $\log (k)$. The wavenumber interval $\Delta k_{n}$ is defined as the following to ensure that the energy at each mode is equal to $E(k) d k$ :

$$
\begin{array}{ll}
\Delta \mathrm{k}_{1}=\left(k_{2}-k_{1}\right) / 2 & \text { for } \mathrm{n}=1 \\
\Delta \mathrm{k}_{\mathrm{n}}=\left(k_{n+1}-k_{n-1}\right) / 2 & \text { for } \mathrm{n} \in\left[2, \mathrm{k}_{\mathrm{N}}-1\right] \\
\Delta \mathrm{k}_{\mathrm{N}}=\left(k_{N_{k}}-k_{N_{k-1}}\right) / 2 & \text { for } \mathrm{n}=\mathrm{k}_{\mathrm{N}}
\end{array}
$$

\subsection{Energy spectrum:}

The fully developed turbulence consists of large number of eddies of different scales; each eddy has a certain kinetic energy. The area between the two boundaries $L_{1}$ and $\eta$ is named "Inertial Range" where the dissipation effect could be neglected. This area is characterized by a power law with an exponent of $-5 / 3$. In this study, we will use an energy spectrum which does not change with time, in the following form

$$
E(k)=\left\{\begin{array}{cc}
C_{k} \varepsilon^{2 / 3} k^{-5 / 3} & k_{l}<k<k_{\eta} \\
0 & \text { otherwise }
\end{array}\right.
$$

$\mathrm{C}_{\mathrm{k}}$ is the Kolmogorov dimensionless constant equal to $\mathrm{C}_{k}=1.5$ (Fung el. al. [6]) and $\varepsilon$ is the dissipation rate of units energy per unit mass. The total kinetic energy, $E$, is obtained by integrating the energy spectrum over the total range of wavenumber as: 


$$
E=\int_{k_{1}}^{k_{N}} E(k) d k
$$

The turbulent velocity fluctuation is:

$$
u^{\prime}=\sqrt{\frac{2}{3} \int_{k_{1}}^{k_{N}} E(k) d k}
$$

where $u^{\prime}$ is the r.m.s. velocity of the turbulent flow.

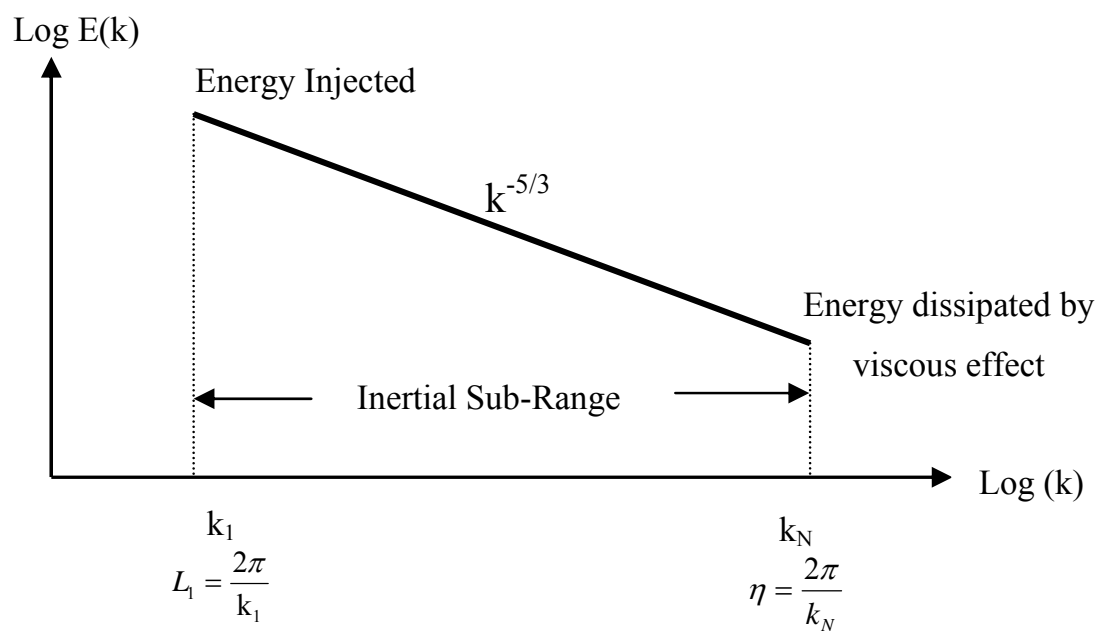

Fig. 1. The Energy Spectrum.

The following definition of the integral length scale of the isotropic turbulence has been used

$$
L=\frac{3 \pi}{4} \frac{\int_{k_{1}}^{k_{N}} k^{-1} E(k) d k}{\int_{k_{1}}^{k_{N}} E(k) d k}
$$

The Kolmogorov length scale is defined as $\eta=\frac{2 \pi}{k_{N}}$. The ratio between the integral length scale to Kolmogorov length scale is $\frac{L_{1}}{\eta}=\frac{\mathrm{k}_{\mathrm{N}}}{k_{1}}$ which is used to determine the inertial range and the associated Reynolds number by the following relation:

$$
\mathrm{Re} \cong\left(\frac{\mathrm{L}_{1}}{\eta}\right)^{4 / 3} \cong\left(\frac{\mathrm{k}_{\mathrm{N}}}{\mathrm{k}_{1}}\right)^{4 / 3}
$$

\subsection{The unsteadiness frequency:}

The frequency $\omega_{n}$ determines the unsteadiness associated with the $n^{\text {th }}$ wavemode. This parameter enables us to create three-dimensional effects in the case of a twodimensional simulation. It has been shown by Malik and Vassilicos [10] that for the first method in three-dimensional isotropic KS for two-particle diffusion, most of the statistical properties are insensitive to the unsteadiness parameter's value provided that it rests in the range $0 \leq \lambda \leq 1$. According to Malik and Vassilicos, the 
unsteadiness frequency $\omega_{n}$ is chosen to be proportional to the eddy-turnover time of the wavemode $\mathrm{n}$ as:

$$
\omega_{\mathrm{n}}=\lambda\left(\mathrm{k}_{\mathrm{n}}{ }^{3} \mathrm{E}\left(\mathrm{k}_{\mathrm{n}}\right)\right)^{1 / 2}
$$

In accordance with these results we have not added any unsteadiness term $(\lambda=0)$ in all KS simulations.

\subsection{Characteristic time scales and computing time step:}

One of the important time scales existing in the turbulent flow is the eddy turnover time scale which corresponds to the integral length scale L. The eddy turnover time is the time needed for the largest eddy to turn around itself. Before this time the particle remembers its initial position, for times larger than the turnover time the particles are free to move randomly.

The turnover time is defined as

$$
t_{d}=\frac{L}{u}
$$

where $L$ is the integral length scale and $u^{\prime}$ is the r.m.s. value of the velocity fluctuation.

The other important time scale is the Kolmogorov time scale which corresponds to the Kolmogorov length scale. The Komogorov time scale is the time needed for a particle to move a distance $\eta$ when its velocity equal to $v_{\eta}$ i.e. $t_{\eta}=\frac{\eta}{v_{\eta}}$ Knowing that $\varepsilon \approx \frac{u^{3}}{L}=\frac{v^{3} \eta}{\eta}$ then

$$
t_{\eta}=t_{d}\left(\frac{\eta}{L}\right)^{2 / 3}
$$

The time step is determined by tracking the motion of fluid element down to the smallest scales; it must be smaller than both the smallest eddy turnover time and the time that a typical fluid particle would take, on average to move by a Kolmogorov length scale. According to Fung [12] a time step equal 0.1 of the Kolmogorov time scale is enough to ensure that the results are independent of the time step within the statistical error.

\section{Heavy Particle Clusters:}

The study of heavy (particles with inertia) multi-particle clusters dispersed in a turbulent flow is important in many environmental, geophysical and industrial processes. The enhancement of concentration in clouds and powders in chemical and pharmaceutical industries or the control of air pollution can be achieved by monitoring the clustering pattern and the concentration fluctuation generated from the interaction of inertial particles with the turbulent flow.

A heavy particle is any small particle in the flow with a density much larger than the density of the fluid carrying it. The resulting mixture is a two-phase flow. Their dynamics of dispersion in a turbulent flow is different from that of fluid elements because of the introduction of additional forces resulting from the relative motion between the particle and the surrounding fluid. During its journey in the flow the particle is subjected to its inertia and to a body force. Therefore, the problem of the dispersion of heavy particles is more difficult to study than that of the diffusion of fluid 
elements as it is characterized by both the properties of the particles (their density and their response time) and that of the turbulent flow which carries the particles.

\subsection{Heavy particle equation of motion:}

Study of the equation of motion of heavy particles is still the subject of much current researchers( Fung [12], Maxey et al [13] and Maxey [14]). Let's consider a heavy particle with its centre positioned at $x_{p}(t)$ and moves with a velocity $v(t)$ in the surrounding flow field of velocity field $u\left(x_{p}, t\right)$. The equation of motion of a heavy spherical particle can be calculated as:

$$
\mathrm{m}_{\mathrm{p}} \frac{\mathrm{dv}}{\mathrm{dt}}=\sum \mathrm{F}_{\text {acting }}(\mathrm{u}, \mathrm{v}, \mathrm{t})
$$

The term on the RHS of equation (13) is the sum of all forces acting on the particle. The equation proposed by Maxey and Riley [13] is the most recent full derivation of the equation of motion for a spherical particle in non-uniform unsteady flow with a particle starting at rest relative to the fluid. Maxey [14] used equation of motion, which is introduced here, without the limitation of the initial velocity of the heavy particle to be equal to fluid velocity. The equation of motion for a spherical heavy particle is written as the follows:

$$
\begin{gathered}
m_{p} \frac{d \mathrm{v}}{d t}=\left(m_{p}-m_{f}\right) g+\left.m_{f} \frac{D u}{D t}\right|_{x_{p}(t)}-\frac{1}{2} m_{f} \frac{d}{d t}\left\{\mathrm{v}(t)-u\left(x_{p}(t), t\right)-\left.\frac{1}{10} a^{2} \nabla^{2} u\right|_{x_{p}(t)}\right\} \\
\left.-6 \pi a \mu H(t)-6 \pi a^{2} \mu \int_{0}^{t} \frac{d H(t)}{d \tau}[\pi v(t-\tau)]^{-1 / 2}\right\} d \tau-6 \pi a^{2} \mu(\pi v t)^{1 / 2} H(0) \\
H(t)=v(t)-u\left(x_{p}(t) . t\right)-\frac{a^{2}}{6} \nabla^{2} u
\end{gathered}
$$

where

and

$m_{p} \quad$ mass of the spherical particle

$m_{f} \quad$ equivalent mass of the displaced fluid

g gravity acceleration

$\mu \quad$ fluid dynamic viscosity

$v \quad$ fluid kinematic viscosity

$x_{p} \quad$ position of the sphere centre which moves with a velocity $v(t)$ through an ambient flow $u(x, t)$.

The different terms in the equation (14) could be explained as follows:

- $\left(m_{p}-m_{f}\right) g$ represents the net body force which represents the cumulative effect of buoyancy force and gravity force. It is based on the difference of density between the heavy particle and the surrounding flow field.

- $\left.m_{f} \frac{D u}{D t}\right|_{x_{p}(t)}-\frac{1}{2} m_{f} \frac{d}{d t}\left\{\mathrm{v}(t)-u\left(x_{p}(t), t\right)-\left.\frac{1}{10} a^{2} \nabla^{2} u\right|_{x_{p}(t)}\right\} \quad$ represents the fluid stress gradient force arising from the pressure and the viscous effects. It is related to the acceleration of the fluid in the vicinity of the particle. Taking into account the effect of the added mass of the fluid which will move with the particle (Elmaihy [15]) 
- $6 \pi a \mu H(t)$ represents the drag force that can be calculated from the relative velocity of the particle and the surrounding fluid and depends on the particle Reynolds number, Rep, based on the particle diameter. The Stokesian drag coefficient (linear drag) for a spherical particle based on the particle Reynolds number is to be less than unity Rep $<1$.

- $\left.6 \pi a^{2} \mu \int_{0}^{t} \frac{d H(t)}{d \tau}[\pi v(t-\tau)]^{-1 / 2}\right\} d \tau$ represents the friction effect in a viscous fluid flow in the transient state known as the Basset history force, this effect results from the diffusion of vorticity of the particle as the particle is moving unsteadily. The past acceleration is included in this effect weighted with $(t-T)^{1 / 2}$, where $(t-T)$ is the time lag since the past acceleration.

- $6 \pi a^{2} \mu(\pi v t)^{1 / 2} H(0)$ is standing for heavy particle having an initial velocity different than that of the surrounding fluid.

\subsection{Assumptions:}

The motion of the heavy spherical particles here is considered to be in a uniform, isotropic, stationary, homogeneous three-dimensional turbulent flow. The fluid has a constant mean velocity taken to be zero for simplicity. The following assumptions have been made in order to simplify the equation of motion of the heavy particle in the turbulent flow:

1. The density of the heavy particle, $\rho_{p}$, is assumed to be much larger than the surrounding fluid density $\rho_{f}$, then the effect of added mass $\left(2^{\text {nd }}\right.$ and $3^{\text {rd }}$ term of the RHS of the equation)and the Basset history term $\left(5^{\text {th }}\right.$ term of the RHS of the equation) may be ignored.

2. Radius of the heavy particle sphere, $a$, is assumed to be smaller than the smallest length scale of turbulence, the Kolmogorov length-scale of turbulence, then the heavy particle responds to all scales of turbulent flow and does not affect the turbulence mechanism.

3. Radius of the spherical heavy particle is considered to be much larger than the free path of fluid molecules then the particle aerodynamic response time is much larger than the mean molecular collision time, and the effect of Brownian motion can be ignored in comparison with the dispersion by turbulence eddies.

4. Radius of the spherical heavy particle is assumed to be sufficiently so small that the Reynolds number based on it be much smaller than unity; then the affecting drag force on the heavy particle may be considered a Stockesian drag (linear Stocks drag),

5. Concentration of particles in the fluid flow field must be small enough to make sure that the interaction between the particles can be ignored so that the presence of the particles does not modify the turbulence flow structure,

6. The relative flow approaching the particle is effectively considered to be uniform so no lift force is introduced, also the domain of turbulence is assumed to be infinite to ignore the interaction between the fluid molecules and solid wall.

Even with all the above-mentioned assumptions, the equation of motion describing the motion of heavy particles in turbulent flows is still under current researches. This equation of motion is still complex and worth studying and is applicable to many 
different problems such as aerosols in gases, small particles in water, and vapour in oil systems.

\subsection{Simplified equation of heavy particle motion:}

After applying the above-mentioned assumptions, the equation of motion of heavy particle which was derived by Maxey [14] can be simplified to achieve our goal and reduce the computational expense. It is considered to be applied on a frame of reference moving with the fluid velocity, as follows:

$$
\begin{gathered}
m_{p} \frac{\mathrm{dv}}{\mathrm{dt}}=\mathrm{m}_{\mathrm{p}} g-6 \pi \mathrm{a} \mu(\mathrm{v}-\mathrm{u}) \\
\frac{\mathrm{dv}}{d t}=\frac{\mathrm{u}-\mathrm{v}+\mathrm{V}_{\mathrm{d}}}{\tau_{\mathrm{a}}}
\end{gathered}
$$

where

$$
\begin{array}{ll}
\tau_{a} & \text { particle's dynamic response time } \\
& \tau_{a}=\frac{\mathrm{m}_{\mathrm{p}}}{6 \pi \mathrm{a} \mu}=\frac{\rho_{\mathrm{p}} d_{p}^{2}}{18 \mu} \\
\mathrm{V}_{\mathrm{d}} & \text { is the Stokes terminal fall velocity or the drift velocity. } \\
& V_{d}=\frac{\mathrm{m}_{\mathrm{p}} g}{6 \pi a \mu}=\tau_{\mathrm{a}} g
\end{array}
$$

Two dimensionless parameters can be introduced:

- The Stokes number which expressed the ratio between the particle's dynamic response time (inertia effect) and the turbulence characteristic time represented in the largest eddy(eddy turn over time scale). It measures the relative importance of the particle inertia. In the limiting case $\mathrm{St}=0$; the inertia particles recover the motion of the fluid particles, on the other hand, at $\mathrm{St}=\infty$, the inertia particles become less and less influenced by the surrounding velocity field.

$$
\mathrm{St}=\tau_{\mathrm{a}} / \mathrm{t}_{\mathrm{d}}
$$

- The drift parameter which is the ratio between the particle's drift velocity and the turbulence rms velocity (turbulence effect):

$$
\mathrm{W}=\mathrm{V}_{\mathrm{d}} / \mathrm{u}
$$

For each simulation, the statistical quantities were obtained by taking the ensemble averaging over 4000 realizations of the flow field. All simulations reported here have been performed using two hundred Fourier modes $(\mathrm{N}=200)$. All statistical quantities are statistically converged with respect to increasing the number of realizations in the ensemble averaging or increasing the number of modes.

\section{Three-Particles Cluster:}

In this section, three-particle cluster parameterization is studied. The evolution of size and shape factors that describe the distortion as a result of dispersion in the isotropic homogenous turbulent flow field is investigated.

\subsection{Three-Particle Cluster parameterization:}

Considering a three-particle cluster locates at $X_{1}, X_{2}$ and $X_{3}$, as shown in Fig.2, the initial positions of the particles are chosen randomly in each realization. The initial 
position of the first particle is chosen randomly, then the other particles positions are calculated relative to the first particle. The initial separation between any two particles is set to be $\Delta_{0}=n \eta$ where $n$ varies from 0.25 to 1000 according to the choice of the inertial sub-range. For more randomness the cube shown in Fig. 2, is rotated by a random angle at eachzealization.

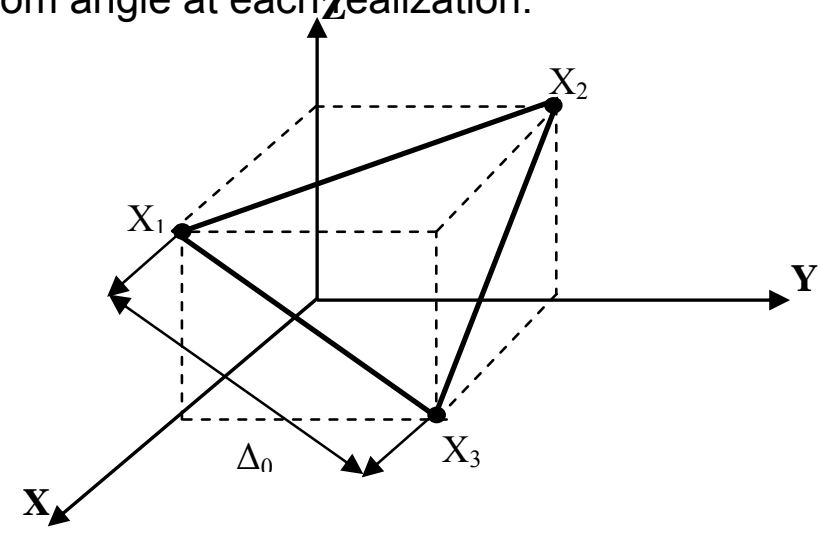

Fig. (2): The initial positions of heavy particle cluster consists of three particles.

The evolution of size and shape of the three-particle cluster corresponding to the points $X_{1}, X_{2}$ and $X_{3}$ is described using the same Eulerian parameterization as that used in (Pumir et. al. [2] and Castiglione and Pumir [3]).

\subsubsection{Size variation parameter:}

A set of two reduced vectors, $\rho_{\mathrm{i}}$, independent of the centre of mass variable $\rho_{0}$ can be defined as follows:

$$
\begin{aligned}
& \vec{\rho}_{1}=\frac{X_{2}-X_{1}}{2 \sqrt{2}} \\
& \vec{\rho}_{2}=\frac{2 X_{3}-X_{1}-X_{2}}{\sqrt{6}}
\end{aligned}
$$

The radius of gyration which is used to characterize the global size of the triangle can be defined as:

$$
R^{2}=\sum_{\mathrm{i}=1}^{2} \vec{\rho}_{\mathrm{i}}^{2}=\overrightarrow{\rho_{1}^{2}}+\overrightarrow{\rho_{2}^{2}}=\frac{\left(\mathrm{r}_{12}^{2}+\mathrm{r}_{23}^{2}+\mathrm{r}_{31}^{2}\right)}{3}
$$

where $r_{i j}=\left|\overrightarrow{\mathrm{X}}_{\mathrm{j}}-\overrightarrow{\mathrm{X}}_{\mathrm{i}}\right|$ are the triangle sides, the triangle area is:

$$
A=\left|\overrightarrow{\rho_{1}} \times \overrightarrow{\rho_{2}}\right|
$$

\subsubsection{Shape variation parameters:}

To characterize the shape of the three-particle object, Pumir el. al. [2] introduced a "moment of inertia-like" tensor as follows:

$$
g^{a b} \equiv \sum_{\mathrm{i}=1}^{2} \rho_{\mathrm{i}}^{\mathrm{a}} \rho_{i}^{b}
$$

where $\rho_{\mathrm{i}}^{\mathrm{a}}$ is the a component of the vector $\rho_{\mathrm{i}}$.

For the three-dimensional velocity fields, there are three eignvalues that describe the spatial extension of the triangle in the three-dimensional turbulence. The triangle 
continuously experience dilatation, rotation and translation during its evolution in the turbulent flow. Its shape is varying continuously; this variation can be calculated through monitoring the value of the quantity $I_{2}$ which is defined as the ratio between $g_{2}$ and $R^{2}$ :

$$
I_{2}=\frac{\mathrm{g}_{2}}{\mathrm{R}^{2}}, 0 \leq \mathrm{I}_{2} \leq 1 / 2
$$

An equilateral triangle corresponds to a value of $I_{2}=1 / 2$, a smaller value of $I_{2}$ corresponds to more elongation of the triangle.

The other method to describe the shape of the triangle is to use the parameters $\mathrm{w}$ and $X$ (Castiglione and Pumir [3]) which are defined as follows:

$$
\begin{gathered}
w=2 \frac{\left\lfloor\overrightarrow{\rho_{1}} \times \overrightarrow{\rho_{2}}\right\rfloor}{\mathrm{R}^{2}} \quad \mathrm{w} \in[-1,1] \\
\chi=\frac{1}{2} \arctan \left[\frac{2 \overrightarrow{\rho_{1}} \cdot \overrightarrow{\rho_{2}}}{\rho_{2}^{2}-\rho_{1}^{2}}\right] \quad \chi \in[0, \pi]
\end{gathered}
$$

The parameter $\mathrm{I}_{2}$ can be related to the parameter $\mathrm{w}$ as:

$$
I_{2}=\left(1-\sqrt{1-w^{2}}\right) / 2
$$

Pumir [16] shows that due to the global invariance of the triangles under any relabelling of the three vertices, the parameter $w \in[-1,1]$ and $\chi \in[0, \pi]$ domain can be reduced to $w \in[0,1], \chi \in[0, \pi / 6]$. The value $\mathrm{w}=0$ indicates that the three points are aligned, whereas the value $w=1$ corresponds to an equilateral triangle.

Small values of $X$ indicate that separation between two particles (e.g 1 and 2 ) is much smaller than their separation with the third one. Fig. 3 shows different examples of the triangle corresponding to different values of $w$ and $X$.
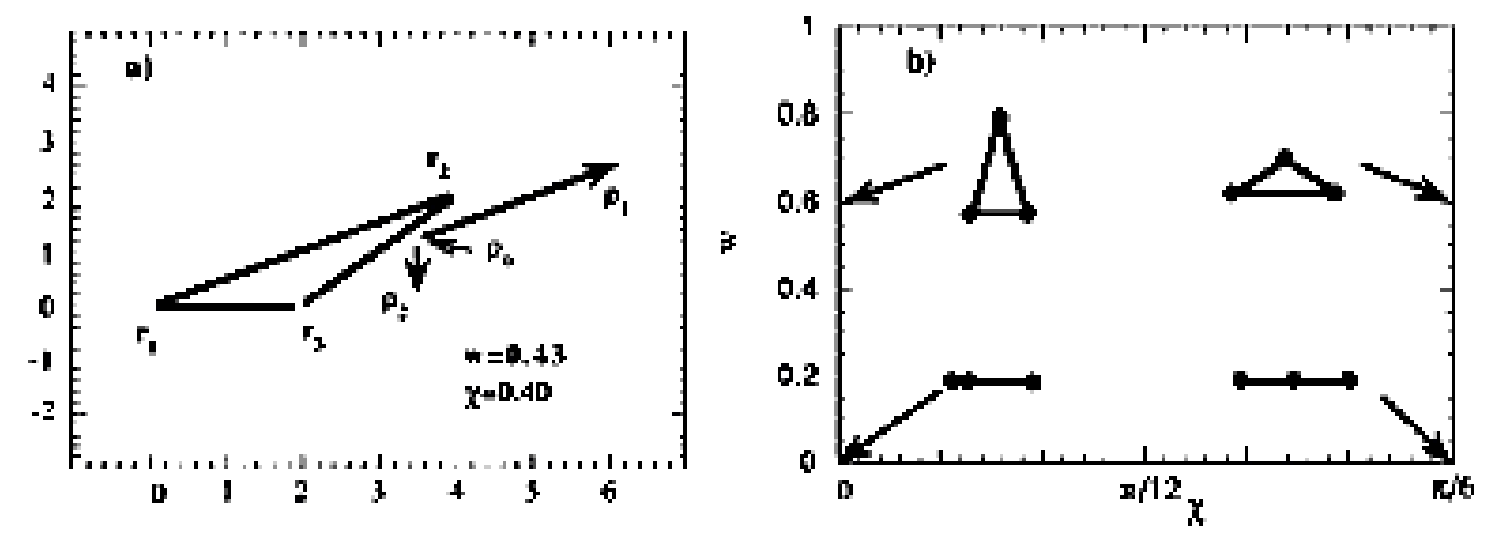

Figure (3): The shape of a triangle as a function of factors $w$ and $X$

(Castiglione and Pumir [3])

\subsection{Three-particle cluster simulation:}

The equations of motions were integrated over 4000 realizations of the flow field and the initial velocity of the heavy particle is set to be the same as the fluid element.

$$
v(t=0)=u\left(x_{p}(t=0), 0\right)+v_{d}
$$

The equations of motion have been integrated using a $4^{\text {th }}$ order Runge-Kutta scheme. The chosen time step has been smaller than the Kolmogorov time length scale in order not to influence the evaluation of the quantities of interest, $R, A, I_{2}, w$, 
and $\mathrm{X}$. The particle time constant, $\mathrm{T}_{\mathrm{a}}$, has been chosen always to be larger than the smallest time scale of the turbulent flow $\left(t_{\eta}\right)$. All runs have been made for $\left(k_{N} / k_{1}\right)=$ 185, 1000 and 2000; to study the effect of higher Reynolds numbers on the inertial sub-ranges with an unsteadiness value of $\lambda=0$.

The ranges of particle inertia parameters, Stokes numbers, $\left(S t=T_{a} / t_{d}\right)$ and fall velocities $\left(\mathrm{W}=\mathrm{V}_{\mathrm{d}} / \mathrm{u}^{\prime}\right)$ are set as follows:

- six values of St, namely $0.05,0.2,0.4,0.6,0.8$ and 1 , are simulated at $W=0$; the first one, $\mathrm{St}=0.05$, is light enough to behave as a fluid element and the last one, $\mathrm{St}=1$, is heavy enough to have significant inertia and "crossingtrajectories" effects (Yudine [17]).

- six values of $\mathrm{W}$, namely $0.2,0.4,0.8,1,2$ and 4 , are simulated at $\mathrm{St}=0.02$ to introduce the "crossing-trajectories" effect.

The initial position of the particle has been chosen randomly in each realization and for each run of the simulation, statistical quantities have been obtained by taking the ensemble average of many realisations; the results have been obtained for 4000 realizations of the velocity field. The number of realisations has been chosen such that the convergence of the results has been fulfilled.

\section{Three-particle cluster results:}

The study of the effect of particle inertia and drift velocity on three-particle clusters at different initial separations and different inertial ranges is presented here. The calculations have been made for three-particle clusters with different inertia (different Stokes numbers) in the absence of gravity and with different drift velocities (different values of $\mathrm{W}$ ) and different initial separations between the three particles.

\subsection{Effect of particle inertia on three-particle cluster:}

The effect of particle inertia (changing Stokes number values) in the absence of gravity $(\mathrm{W}=0)$ on the dispersion of a three-particle cluster in a homogenous isotropic turbulent flow has been studied. The evolutions of size and shape of an initially equilateral triangle immersed in isotropic turbulence are presented. The size of the triangle is monitored as a function of time by computing the evolution of $\left\langle R^{2}\right\rangle^{1 / 2}$, where $R$ is the radius of gyration, for different initial triangle sizes. The changes in triangle's shape are measured using the parameters $\langle w\rangle,\left\langle I_{2}\right\rangle$ and $\langle x\rangle$ as functions of time.

\subsubsection{Comparison with diffusion case:}

To compare the dispersion of the heavy particle clusters in turbulent flow and the diffusion of fluid particle cluster, both cases have been run under the same conditions. The diffusion process has been run for the three-particle cluster for an inertial sub-range of $\mathrm{k}_{N} / \mathrm{k}_{1}=185$ and initial separations $\Delta_{0} / \eta=0.09,0.25$ and $\Delta_{0} / \eta=4$. The dispersion process has been also run for the same conditions with a Stokes number $\mathrm{St}=1$ and no gravity (drift velocity). Fig. 4, presents the time evolution of the triangle size $\left\langle R^{2}>^{1 / 2}\right.$ and shape factor $\langle W\rangle$ as a function of the time normalized by the largest eddy turnover time for an inertial sub-range $k_{N} / k_{1}=185$ and an initial separation $\Delta_{0} / \eta=4$ for both cases: diffusion of marked fluid elements and dispersion of heavy particles. Fig.5 presents the time evolution of the triangle size and shape factor $\langle\chi\rangle$ as a function of the time normalized by the largest eddy turnover time for an inertial sub-range $\mathrm{k}_{N} / \mathrm{k}_{1}=185$ and an initial separation $\Delta_{0} / \eta=0.25$ for both cases: diffusion of marked fluid elements and dispersion of heavy particles.

Fig. 6 , presents the time evolution of the triangle size and shape factor $\langle\chi\rangle$ as a function of the time normalized by the largest eddy turnover time for an inertial sub- 
range $k_{N} / k_{1}=185$ and an initial separation $\Delta_{0} / \eta=0.09$ for both cases: diffusion of marked fluid elements and dispersion of heavy particles.
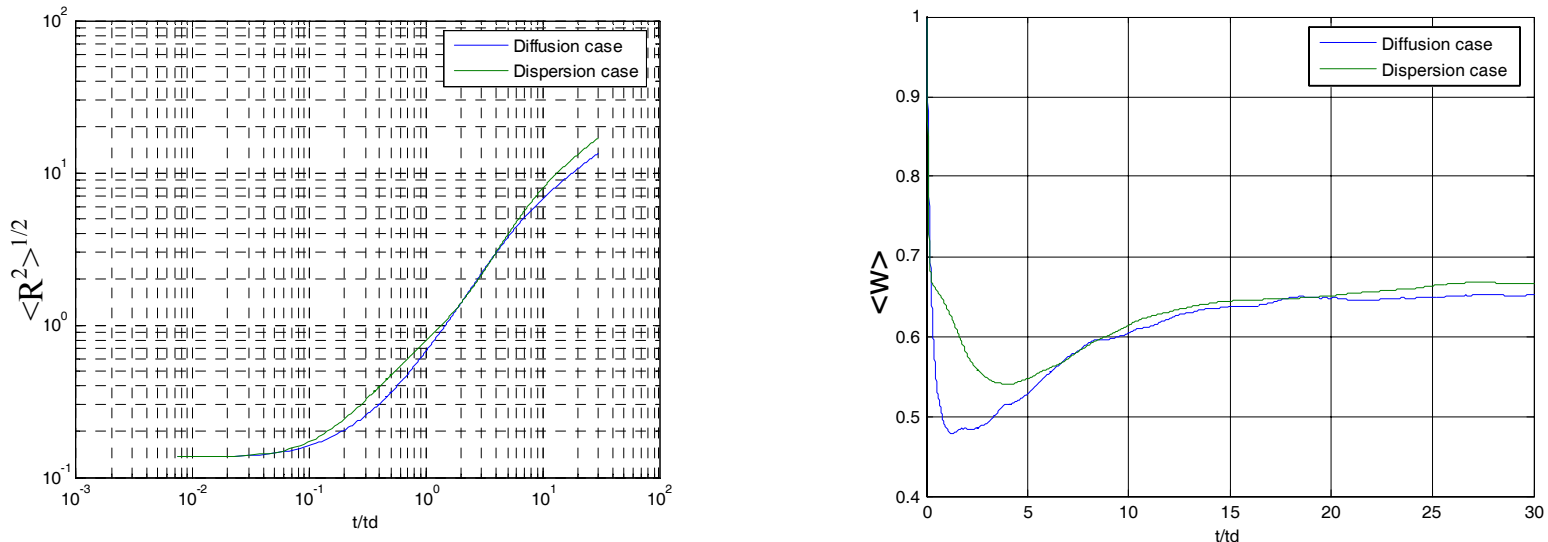

Fig.4. Time evolution of the triangle size and shape factor $\langle\mathrm{W}\rangle$ for $\mathrm{k}_{\mathrm{N}} / \mathrm{k}_{1}=185$ and an initial separation $\Delta_{0} / \eta=4$ for both diffusion and dispersion of heavy particles.

( $\mathrm{St}=1$ and zero drift velocity)
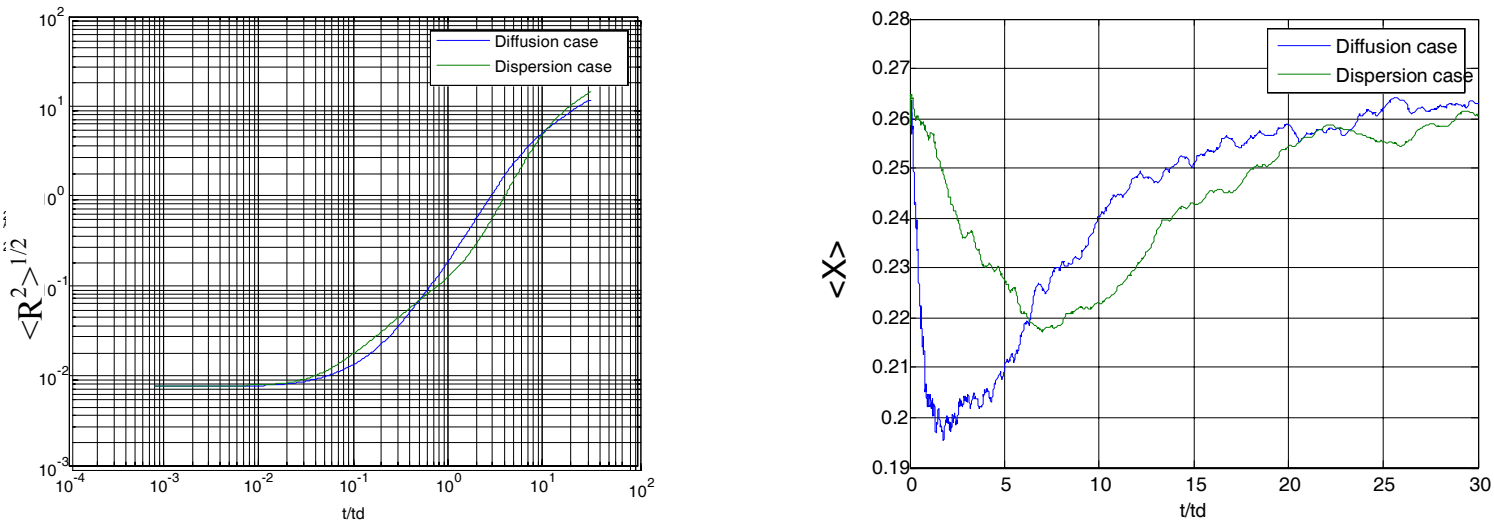

Fig.5. Time evolution of the triangle size and shape factor $\langle\chi\rangle$ for $k_{N} / k_{1}=185$ and an initial separation $\Delta_{0} / \eta=0.25$ for both diffusion and dispersion of heavy particles.

( $\mathrm{St}=1$ and zero drift velocity)
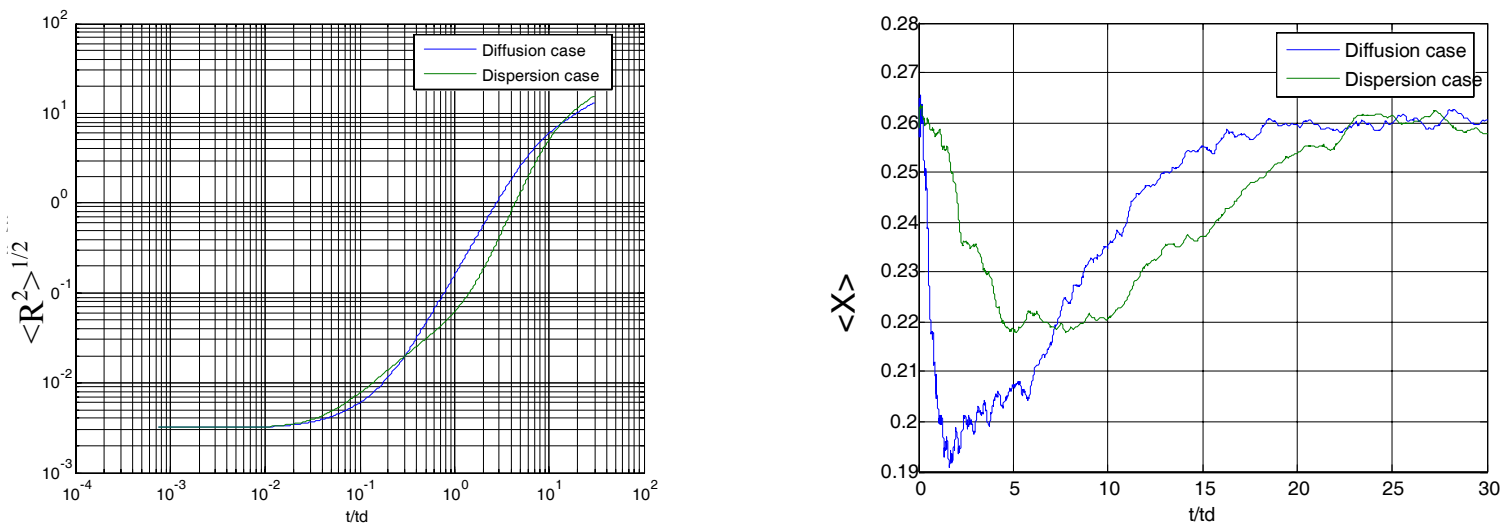

Fig.6. Time evolution of the triangle size and shape factor $\langle\chi\rangle$ for $k_{N} / k_{1}=185$ and an initial separation $\Delta_{0} / \eta=0.09$ for both diffusion and dispersion of particles.

(St $=1$ and zero drift velocity) 
The size and shape of the three-particle cluster in turbulent flow is affected by the dispersion process in which the heavy particles are released over the diffusion process as follows: related to the size of the cluster, it can be shown that if the initial separation is above the Kolmogorov length-scale, the dispersion process is larger than the diffusion process in the turbulent flow. But if the initial separation is below the Kolmogorov length-scale, there will be a critical time before which the dispersion process is more important and after which the diffusion process is more significant. This critical time decreases when the initial separation decreases. The same trend can be noticed on the shape of the cluster.

\subsubsection{Effect of varying initial separation at the same Stokes number:}

In this section, we study the effect of the initial separation on the dispersion of a three-particle cluster in a homogenous isotropic turbulent flow. This is done for a given particle inertia (Stokes number) and a zero drift velocity. The evolution of the size and the geometry of an initially equilateral triangle are presented.

Fig. 7 , presents the evolution of the triangle size and shape factor $\langle w\rangle$ as a function of the time normalized by the largest eddy turnover time for an inertial sub-range $\mathrm{k}_{\mathrm{N}} / \mathrm{k}_{1}=185$, a Stokes number $\mathrm{St}=1$ and a zero drift velocity for different initial separations $\Delta_{0} / \eta=0.25,1,4,16,32,64$ and 92.5 .
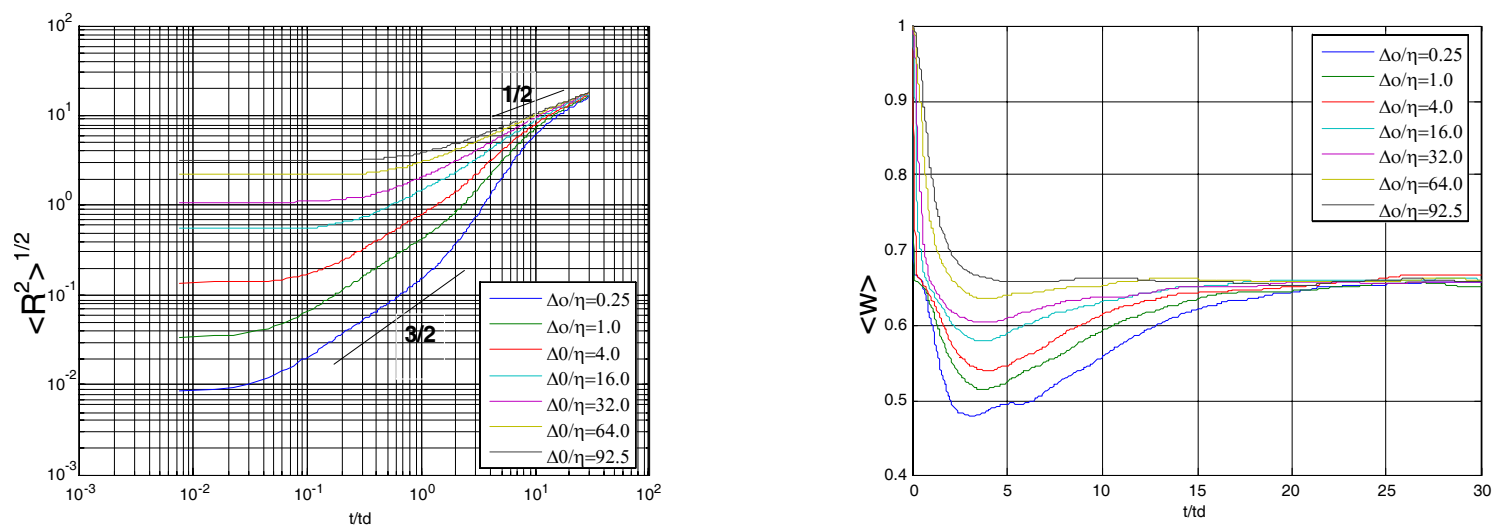

Fig.7). Time evolution of the triangle size and shape factor $\langle\chi\rangle$ for $\mathrm{k}_{N} / \mathrm{k}_{1}=185$, St $=1$ and $W=0$ for different initial separations: $\Delta_{0} / \eta=0.25,1,4,16,32,64$ and 92.5
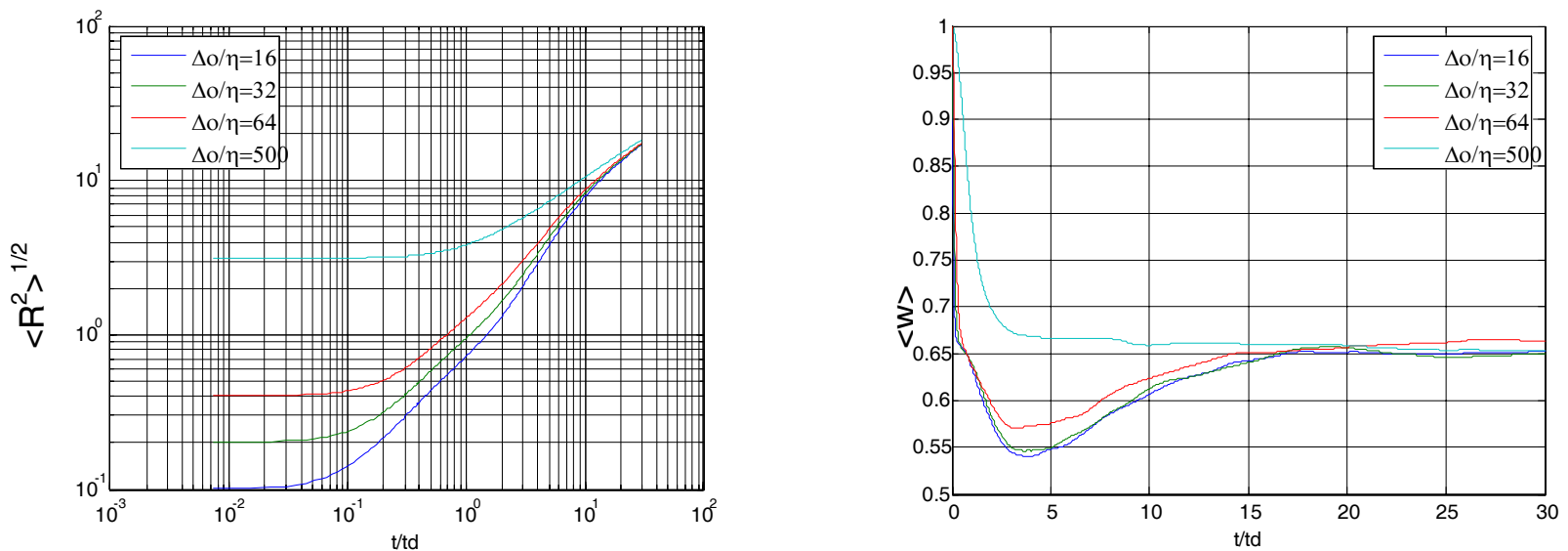

Fig.8. Time evolution of the triangle size and shape factor $\langle W\rangle$ for $k_{N} / k_{1}=1000, S t=$ 1 and $W=0$ for different initial separations: $\Delta_{0} / \eta=16,32,64$ and 500 . 
Fig. 8, presents the evolution of the triangle size and shape factor $\langle w\rangle$ as a function of time normalized by the largest eddy turnover time for an inertial sub-range $\mathrm{k}_{\mathrm{N}} / \mathrm{k}_{1}=1000$, a Stokes number $\mathrm{St}=1$ and a zero drift velocity for different initial separations $\Delta_{0} / \eta=16,32,64$ and 500 .

For the size factor at the same Stokes number, one can observe that with the increase of initial separation the size reaches the largest length scale and is going towards the asymptotic values without experiencing the Richardson regime. For the shape factor at a constant Stokes number, it can be noticed that the minimum value of the factors $\mathrm{w}, \mathrm{I}_{2}$ and $\chi$ is decreased with increase of initial separation, by continuing the increase of the initial separation these parameters reach the asymptotic value directly without passing through a minimum value.

According to Pumir et al [2], at large time all curves corresponding to different initial sizes approach an asymptotic value $\langle\mathrm{W}\rangle_{\text {asy }}=0.5,\left\langle\mathrm{I}_{2}\right\rangle_{\text {asy }}=0.11$ and $\langle\chi\rangle_{\text {asy }}=0.25$ while their Gaussian values are $\langle W\rangle_{\text {Gau }}=1 / 2,\left\langle I_{2}\right\rangle_{\text {Gau }}=0.107$ and $\langle\chi\rangle_{\text {Gau }}=0.262$, these values have also been obtained for the diffusion case studied by Nicolleau and EIMaihy [5] at large time and they obtained these values 0.66 for $\langle w\rangle, 0.16$ for $\left\langle l_{2}\right\rangle$ and 0.26 for $\langle x>$.

We can extend the validity of values obtained by Nicolleau and EIMaihy [5] for the dispersion of heavy particle clusters to the case of varying Stokes number at zero drift velocity.

With the increase of initial separation between heavy particles in the clusters, the minimum value of shape factor $w$ is increased as shown in Fig. 9.

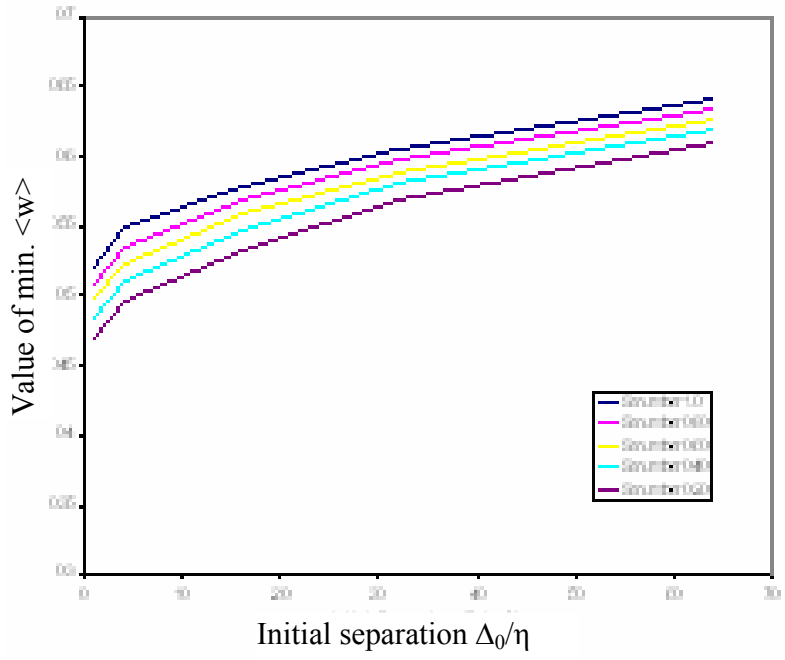

Fig.9. Minimum values of the triangle shape factor $\langle w\rangle$, for different initial separations $\left(\Delta_{0} / \eta=1,4,16,32\right.$ and 64$)$, different Stokes numbers $(\mathrm{St}=0.2,0.4,0.6$, 0.8 and 1.0 from bottom to top) and zero drift velocity, for $k_{N} / k_{1}=185$

\subsubsection{Effect of varying Stokes number at the same initial separation:}

In this section, the effect of particle inertia on the dispersion of three-particle cluster in a homogenous isotropic turbulent flow is studied for an initial separation and zero drift velocity. The evolution of the size and the geometry of an initially equilateral triangle immersed in an isotropic turbulent flow are presented.

Fig.10, presents the evolution of the triangle size as function of time normalized by the largest eddy turnover time for an initial separation $\Delta_{0} / \eta=16$, a zero drift velocity, 
an inertial sub-range $\mathrm{k}_{\mathrm{N}} / \mathrm{k}_{1}=185$ and at different Stokes numbers $\mathrm{St}=0.2,0.4,0.6,0.8$ and 1.
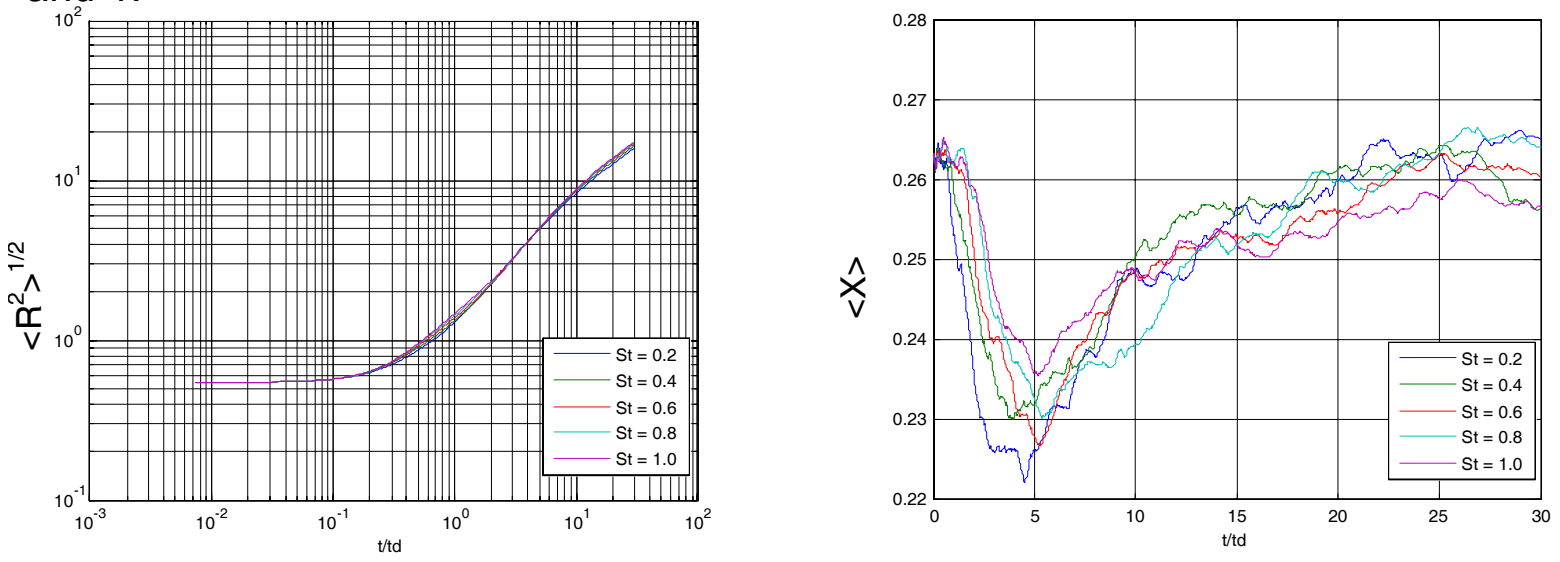

Fig.10. Time evolution of the triangle size and shape factor $\langle\chi\rangle$ for $k_{N} / k_{1}=185$, an initial separation $\Delta_{0} / \eta=16$ and zero drift velocity at different Stokes number $(\mathrm{St}=0.2,0.4,0.6,0.8$ and 1$)$

It can be seen that with the increase of inertia (Stokes number) at a fixed initial separation between the heavy particles in the clusters, the minimum value of the shape factor $\langle w\rangle$ increased. Consequently, they tend to reach the asymptotic value faster than in case of diffusion, this is illustrated in Fig. 11.

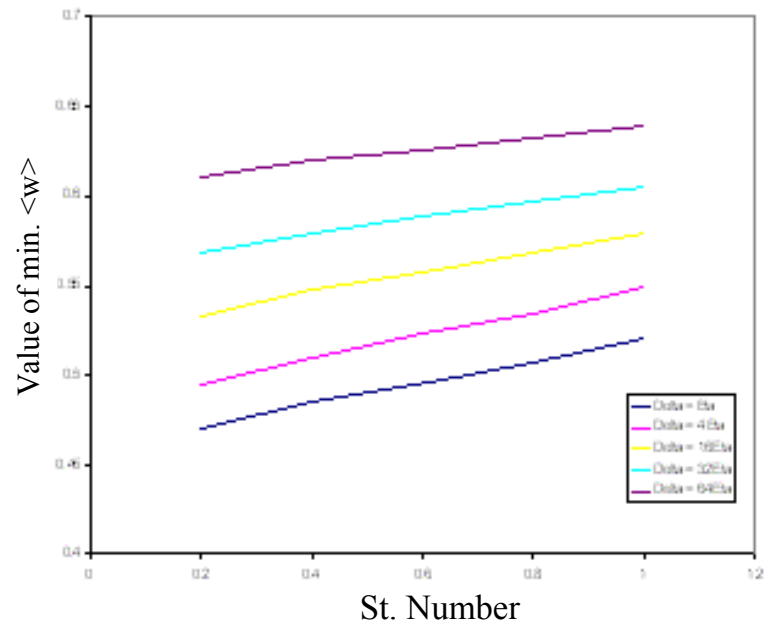

Fig.11. Minimum values of the triangle shape factor $\langle w\rangle$, at different initial separations $\left(\Delta_{0} / \eta=1,4,16,32\right.$, and 64$)$, different Stokes numbers $(S t=0.2,0.4,0.6,0.8$ and 1.0$)$ and zero drift velocity, for $\mathrm{k}_{\mathrm{N}} / \mathrm{k}_{1}=185$.

\subsubsection{Effect of Reynolds number in presence of inertia effect:}

Here the obtained results are plotted for different Reynolds numbers at certain Stokes number and for initial separations between the heavy particles in the clusters either less than the Kolmogorov length-scale or more than the Kolmogorov length-scale. Fig. 12, presents the evolution of the triangle size and shape factor $\langle w\rangle$ as function of time normalized by the eddy turnover time for Stokes numbers $S t=1$, zero drift velocity and initial separation $\Delta_{0} / L_{1}=0.0005$ (below the Kolmogorov length scale), and at different inertial sub-range of $k_{N} / k_{1}=185,1000$ and 2000. Fig.13, presents the evolution of the triangle size and shape factor $\langle w>$ as function of the time normalized by the eddy 
turnover time for a Stokes numbers St=1, zero drift velocity and initial separation $\Delta_{0} / L_{1}=$ 0.032 (above the Kolmogorov length scale), and for different inertial sub-range of $\mathrm{k}_{\mathrm{N}} / \mathrm{k}_{1}=185,1000$ and 2000 . Fig.14, presents the evolution of the triangle size and shape factor $\langle w\rangle$ as function of time normalized by the eddy turnover time for a Stokes numbers $S t=1$, zero drift velocity and initial separation $\Delta_{0} / L_{1}=0.5$ (above the Kolmogorov length scale), and at different inertial sub-ranges $\mathrm{k}_{N} / \mathrm{k}_{1}=185,1000$ and 2000 .
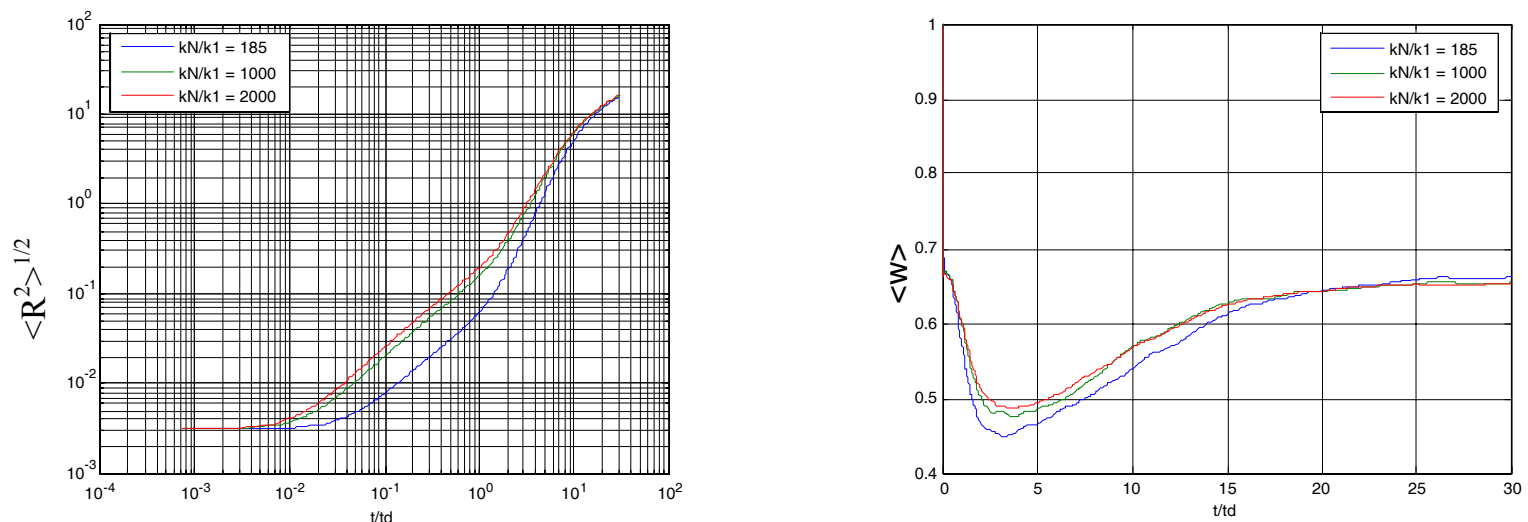

Fig.12. Time evolution of the triangle size for the initial separation $\Delta_{0} / L_{1}=0.0005$, St $=1$ and zero drift velocity for different Reynolds numbers

$$
\mathrm{k}_{N} / \mathrm{k}_{1}=185\left(\Delta_{0} / \eta=0.09\right), 1000\left(\Delta_{0} / \eta=0.5\right) \text { and } 2000\left(\Delta_{0} / \eta=1\right)
$$
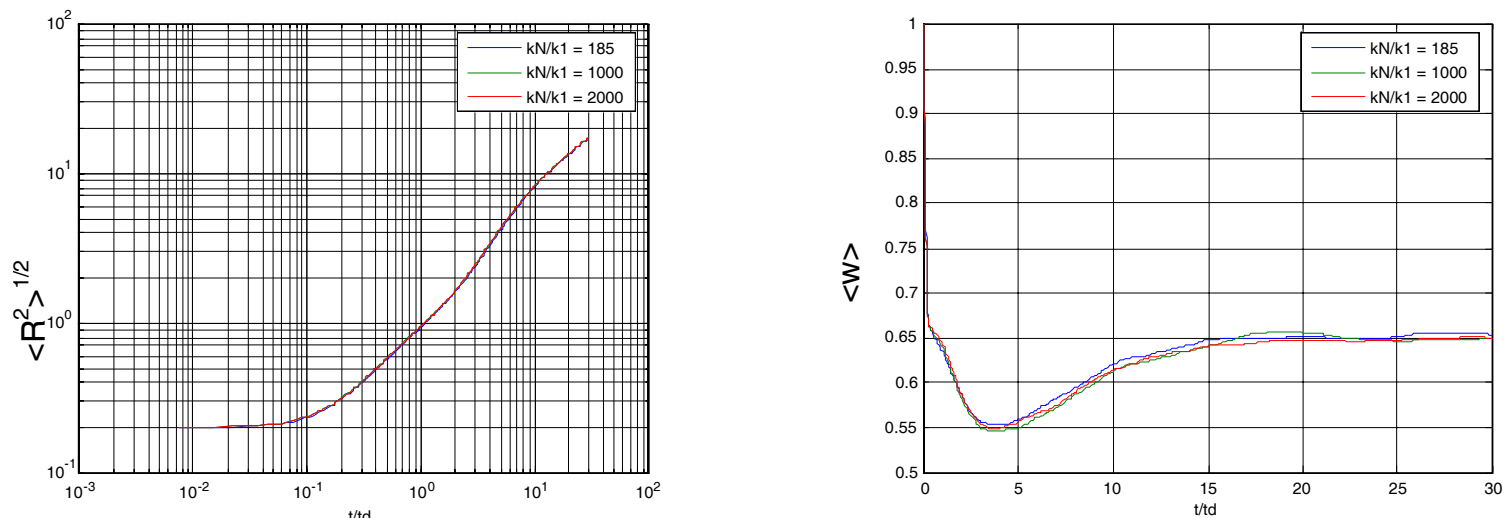

Fig.13. Time evolution of the triangle size for the initial separation $\Delta_{0} / \mathrm{L}_{1}=0.032$, St $=1$ and zero drift velocity for different Reynolds numbers $\mathrm{k}_{\mathrm{N}} / \mathrm{k}_{1}=185\left(\Delta_{0} / \eta=6\right), 1000\left(\Delta_{0} / \eta=32\right)$ and $2000\left(\Delta_{0} / \eta=64\right)$
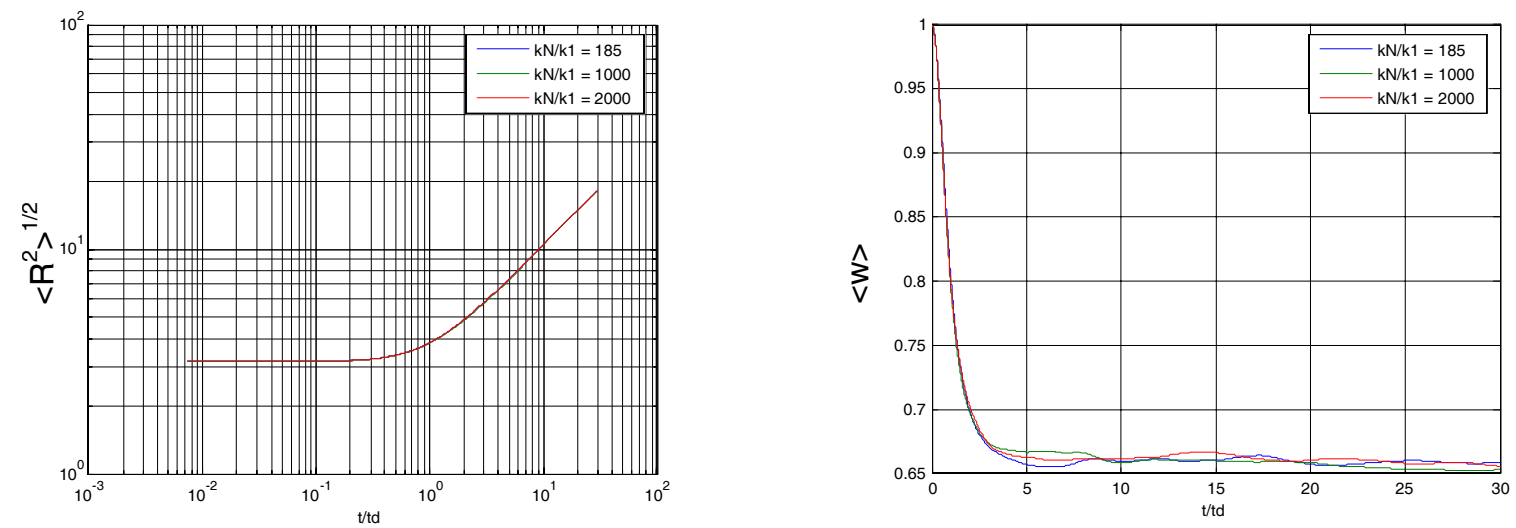
Fig.14. Time evolution of the triangle size for the initial separation $\Delta_{0} / L_{1}=0.5, S t=1$ and zero drift velocity for different Reynolds numbers

$$
\mathrm{k}_{N} / \mathrm{k}_{1}=185\left(\Delta_{0} / \eta=92.5\right), 1000\left(\Delta_{0} / \eta=500\right) \text { and } 2000\left(\Delta_{0} / \eta=1000\right)
$$

All curves for constant value of $\Delta_{0} / L_{1}=0.032$ and $\Delta_{0} / L_{1}=0.5$ are approximately collapsed for the plotted values of $\langle\mathrm{R}\rangle^{1 / 2},\langle\mathrm{~W}\rangle$ and $\langle\chi\rangle$. This result was obtained in the case of cluster diffusion (Nicolleau and EIMaihy [5]). We extend their findings to the heavy particle dispersion for Stokes numbers in the range of $0.2<\mathrm{St}<1$ at zero drift velocity for the range of inertial ranges $185<\mathrm{k}_{N} / \mathrm{k}_{1}<2000$.

It is also noticed that as the initial triangle size increases the shape distortion becomes weaker. When the initial triangle size becomes comparable to the largest length scale $L_{1}\left(\Delta_{0} \approx 0.5 L_{1}\right)$, we find that the parameters describing the shape change decrease from their initial values to their asymptotic values directly without passing through a minimum value and then increase to the asymptotic values as seen for smaller initial triangle sizes $\left(\Delta_{0}<<0.5 \mathrm{~L}_{1}\right)$.

For the initial separation between the heavy particles below the Kolmogorov lengthscale, $\Delta_{0} / L_{1}=0.005$, the curves of $\langle R\rangle^{1 / 2},\langle\mathrm{~W}\rangle$ and $\langle\chi\rangle$ do not collapse. So, we limit the above conclusion to initial separations only above the kolmogorov length-scale.

\subsection{Effect of particle drift-velocity on three-particle cluster:}

In this section, the effect of the particle drift velocity (changing the drift parameter W) in the presence of particle inertia (Stokes number is fixed to the value of 0.02) on the dispersion of three-particle cluster in a homogenous isotropic turbulent flow is studied. The evolution of the size and geometry of an initially equilateral triangle dispersed in isotropic turbulent flow is presented.

\subsubsection{Comparison with diffusion case:}

Here, a direct comparison with the diffusion of the three-particle cluster has been introduced over the dispersion of the heavy particle clusters in the turbulent flow. Both processes will be run at the same conditions. The running conditions are for an inertial sub-range of $\mathrm{k}_{\mathrm{N}} / \mathrm{k}_{1}=185$ and with an initial separation $\Delta_{0} / \eta=0.25$ (below the Kolmogorov length scale) and for $\Delta_{0} / \eta=4$ (above the Kolmogorov length scale). In the dispersion process, heavy particle parameters will be for inertia effect Stokes number $S t=0.02$ and for gravity effect $W=0.2,1$ and 2 .

Fig. 15, presents the evolution of the triangle size and shape factor $\langle\chi\rangle$ as function of time normalized by the largest eddy turnover time at inertial sub-range of $\mathrm{k}_{N} / \mathrm{k}_{1}=185$, initial separation $\Delta_{0} / \eta=0.25$ for both cases of diffusion of marked fluid element and dispersion of heavy particle clusters.

Fig.16, presents the evolution of the triangle size and shape factor $\langle\chi\rangle$ as function of time normalized by the largest eddy turnover time at inertial sub-range of $k_{N} / k_{1}=185$, initial separation $\Delta_{0} / \eta=4$ for both cases of diffusion of marked fluid element and dispersion of heavy particle clusters. The size and shape of three-particle cluster in the turbulent flow could be affected by the dispersion process in which the heavy particles are released over the diffusion process as follows: Related to the size of cluster, with the increase of drift velocity, whether the initial separation is below or above the Kolmogorov length scale, the dispersion process enhances the size distortion of the cluster by reaching the asymptotic value faster than the diffusion process. Related to the shape of the cluster, also with the increase of drift velocity whether the initial separation is below or above the Kolmogorov length scale the dispersion helps the cluster to reach the minimum value faster than the diffusion. In the dispersion case it can be noticed that the asymptotic value are obtained as 
$\left\langle\mathrm{I}_{2}\right\rangle_{\text {asy }}=0.152,\langle\mathrm{w}\rangle_{\text {asy }}=0.632$ which are lower than the obtained value in the diffusion case $\left(\left\langle\left.\right|_{2}\right\rangle_{\text {asy }}=0.16,\langle w\rangle_{\text {asy }}=0.66\right)$ while $\langle\chi\rangle_{\text {asy }}$ is as the same in the diffusion case which and $(<\chi>$ asy= 0.26); Nicolleau and EIMaihy [5]).
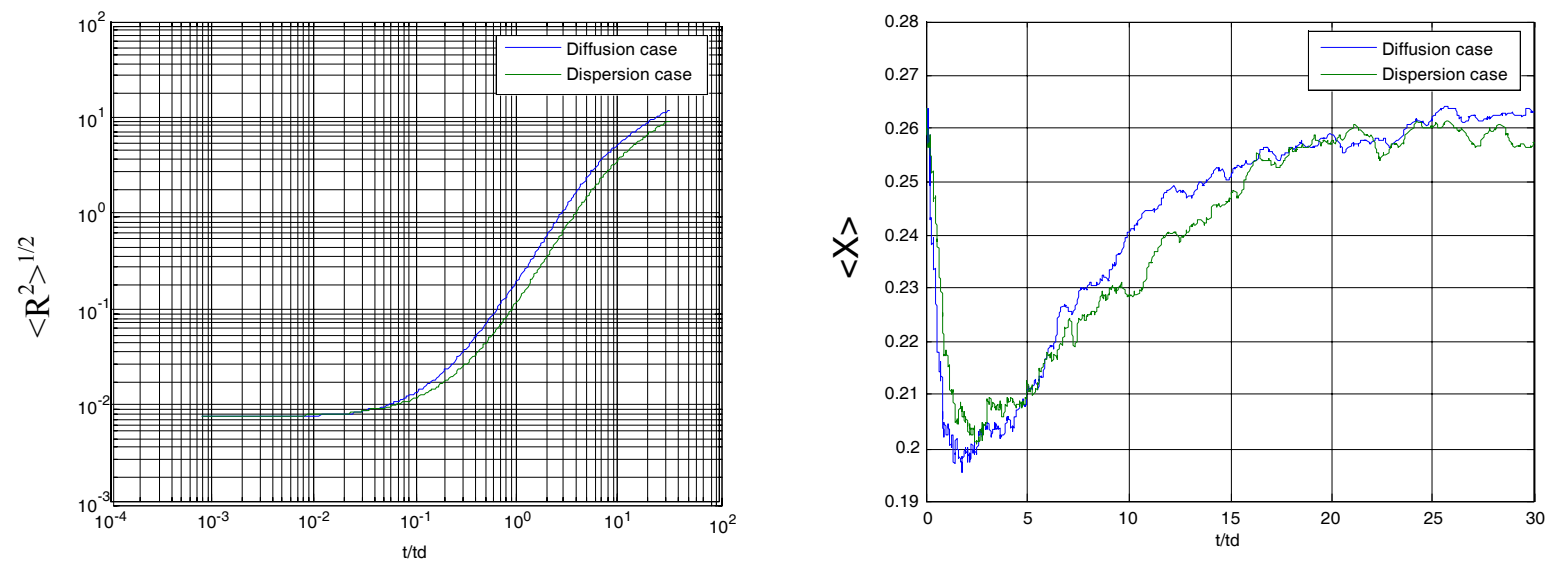

Fig.15. Time evolution of the triangle size and shape factor $\langle\chi\rangle$ for $k_{N} / k_{1}=185$ and an initial separation $\Delta_{0} / \eta=0.25$ for both cases: diffusion and dispersion of heavy particles

$(\mathrm{St}=0.02$ and $\mathrm{W}=2)$
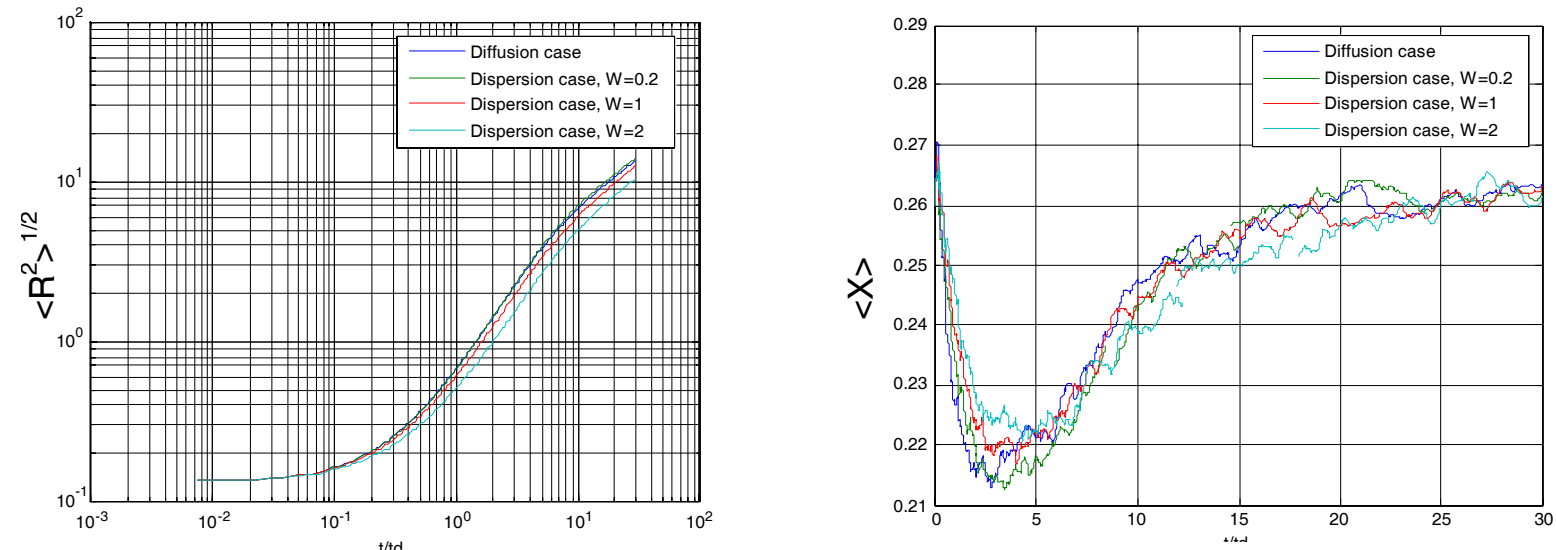

Fig.16. Time evolution of the triangle size and shape factor $\langle\chi\rangle$ for $k_{N} / k_{1}=185$ and an initial separation $\Delta_{0} / \eta=4$ for both cases: diffusion and dispersion of heavy particles (St $=0.02$ and different drift velocity $\mathrm{W}=0.2,1$ and 2 )

\subsubsection{Effect of varying initial separation at same drift velocity:}

In this section, we study the effect of initial separation on the dispersion of threeparticle cluster in a homogenous isotropic turbulent flow. This is done for a given drift velocity at a fixed particle inertia (Stokes number). The evolution of the size and geometry of an initially equilateral triangle dispersed in isotropic turbulent flow are presented.

Fig.17. presents the evolution of the triangle size and shape factor $\langle w\rangle$ as function of time normalized by the largest eddy turnover time for an inertial sub-range $\mathrm{k}_{\mathrm{N}} / \mathrm{k}_{1}=185$, Stokes number $\mathrm{St}=0.02$ and drift velocity $\mathrm{w}=0.2$ for different initial separations $\Delta_{0} / \eta=4,16,32,64$ and 92.5 . 
It can be noticed that with the increase of initial separation, the size reaches the largest length scale and is going towards the asymptotic values without experiencing the Richardson regime. Also for the shape factor at constant drift velocity and Stokes number, it is noticed that the minimum value of the factors $\mathrm{w}, \mathrm{I}_{2}$ and $\chi$ is decrease with the increase of initial separation. These parameters reach asymptotic value directly without passing through a minimum value by continuing the increase of the initial separation.
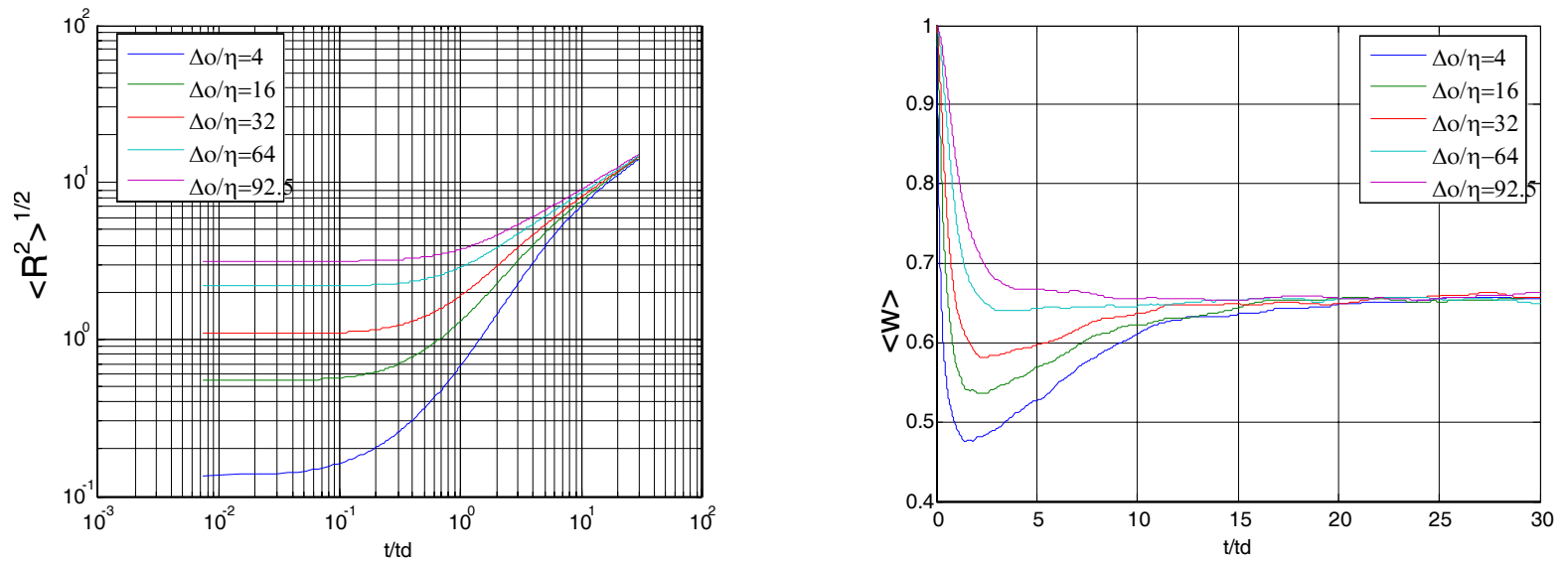

Fig.17. Time evolution of the triangle size and shape factor $<w>$ for $k_{N} / k_{1}=185$, St $=$ 0.02 and drift velocity $W=0.2$ at different initial separations $\Delta_{0} / \eta=4,16,32,64$ and

\section{5}

\subsubsection{Effect of varying drift velocity at the same initial separation:}

In this section, the evolution of the size and geometry of initially equilateral triangle disperse in isotropic turbulent flow are studied for a given drift velocity at a fixed particle inertia (Stokes number).

Fig.18, presents the evolution of the triangle size and shape factor $\langle w\rangle$ as function of time normalized by the eddy turnover time are plotted for inertial sub-range $\mathrm{k}_{\mathrm{N}} / \mathrm{k}_{1}=185$ for Stokes number $\mathrm{St}=0.02$ and initial separation $\Delta_{0} / \eta=92.5$ at different drift velocity parameters $W=0.2,0.8,1,2$ and 4 .

Fig.19, presents the evolution of the triangle size and shape factor $\langle w\rangle$ as a function of time normalized by the eddy turnover time is plotted for inertial sub-range $\mathrm{k}_{\mathrm{N}} / \mathrm{k}_{1}=1000$, Stokes number $\mathrm{St}=0.02$ and initial separation $\Delta_{0} / \eta=92.5$ at different drift velocity parameters $W=0.2,0.8,1,2$ and 4 .
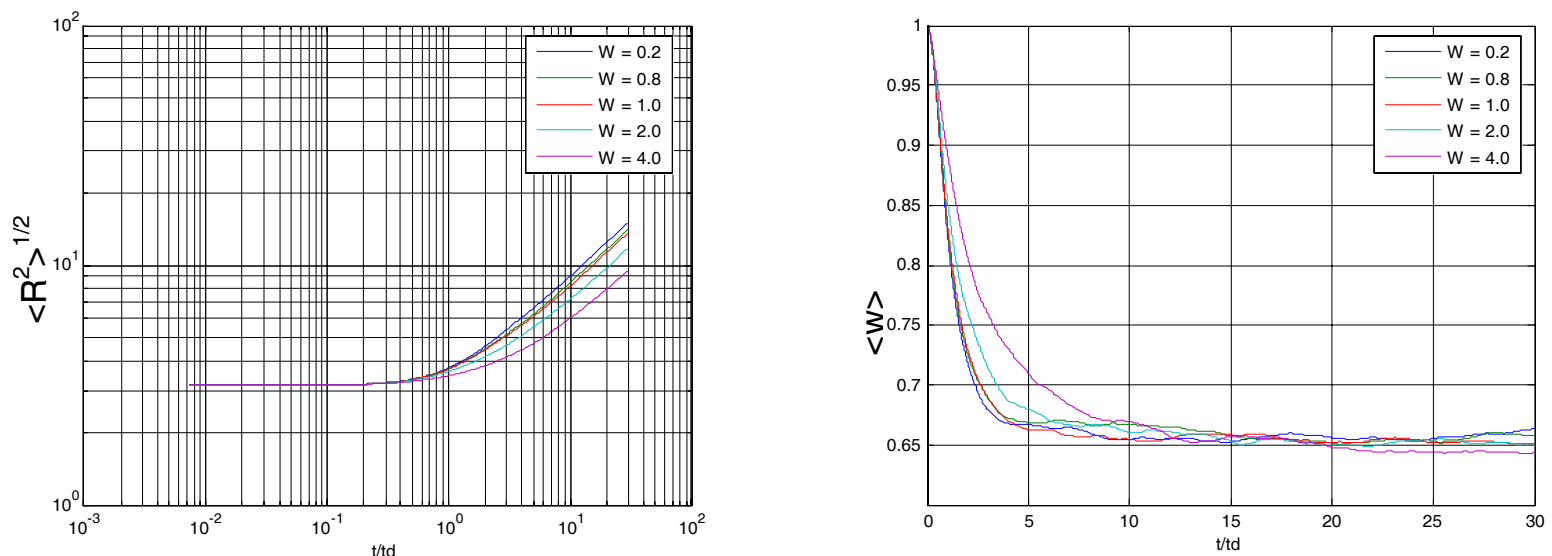
Fig. 18. Time evolution of the triangle size and shape factor $<w>$ for $k_{N} / k_{1}=185$, initial separation $\Delta_{0} / \eta=92.5$ and $S t=0.02$ at different drift velocity parameters $\mathrm{W}=0.2$,

$0.8,1,2$ and 4
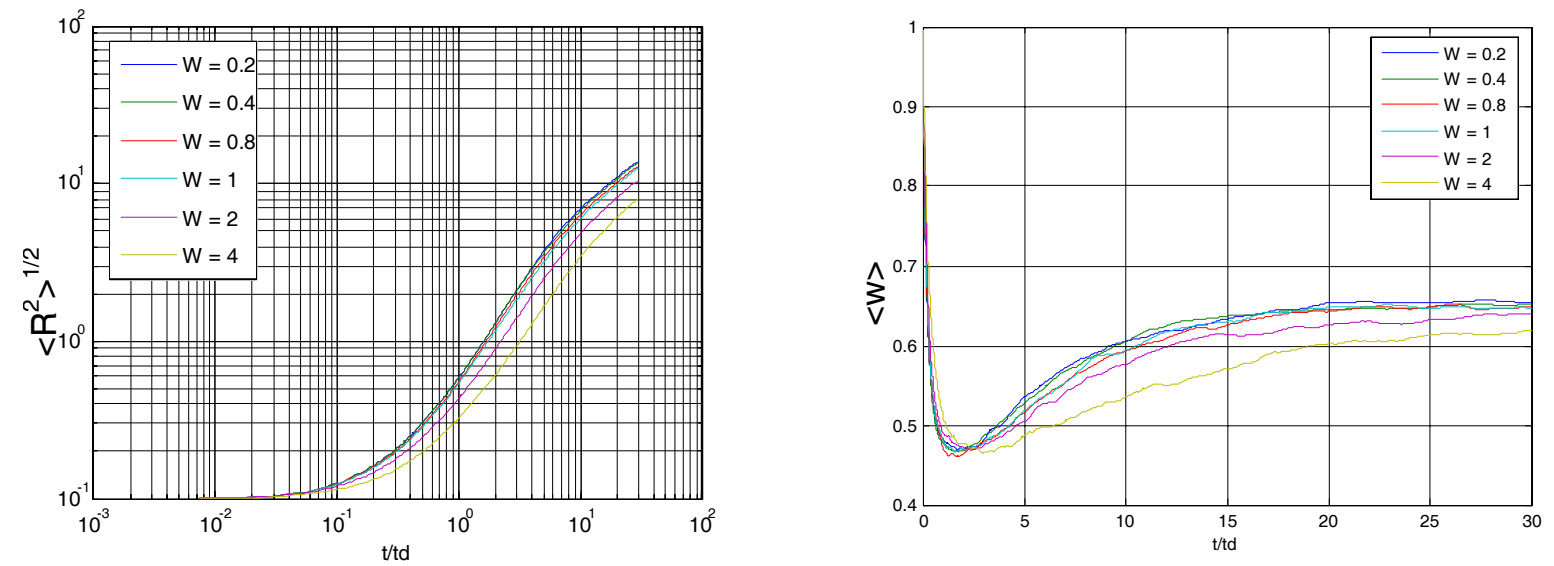

Fig.19. Time evolution of the triangle size and shape factor $<w>$ for $k_{N} / k_{1}=1000$, initial separation $\Delta_{0} / \eta=16, \mathrm{St}=0.02$ at different drift velocity parameter $\mathrm{W}=0.2,0.4,0.8,1,2$ and 4

With the increase of drift velocity parameter at a fixed initial separation between the heavy particles in the clusters, the shape factor $W$ minimum value is the same but the asymptotic value which it reaches is becoming lower. As a consequence, they tend to reach lower asymptotic value faster than in the case of the diffusion.

5.2.4 Effect of Reynolds number in presence of drift-velocity:

Here the results obtained are plotted for different Reynolds number at certain Stokes number for initial separation $\Delta_{0} / L_{1}=0.5$ and $\Delta_{0} / L_{1}=0.032$.

Fig.20, presents the evolution of the triangle size and shape factor $\langle w\rangle$ as function of time normalized by the eddy turnover time are plotted at Stokes numbers $\mathrm{St}=$ 0.02 , drift velocity parameter $W=0.2$, and an initial separation $\Delta_{0} / L_{1}=0.032$ at different inertial sub-ranges of $\mathrm{k}_{\mathrm{N}} / \mathrm{k}_{1}=185,1000$ and 2000.

Fig. 21, presents the evolution of the triangle size and shape factor $\langle\mathrm{w}\rangle$ as function of time normalized by the eddy turnover time are plotted at Stokes numbers $\mathrm{St}=0.02$ ,drift velocity parameter $W=0.2$ and an initial separation $\Delta_{0} / L_{1}=0.5$ at different inertial sub-ranges of $\mathrm{k}_{\mathrm{N}} / \mathrm{k}_{1}=185,1000$ and 2000.
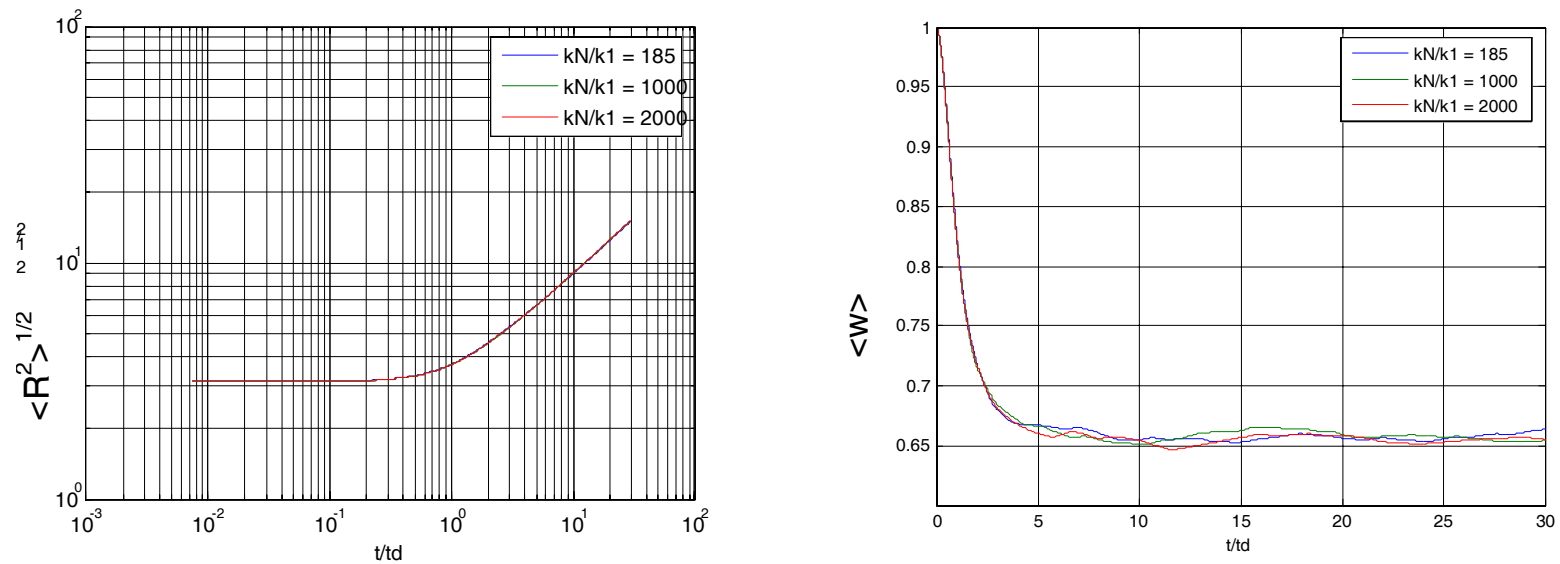
Fig.20. Time evolution of the triangle size and shape factor $\langle W\rangle$ for initial separation $\Delta_{0} / L_{1}=0.032, S t=0.02$ and drift velocity parameter $\mathrm{W}=0.2$ at different Reynolds numbers $k_{N} / k_{1}=185\left(\Delta_{0} / \eta=6\right), 1000\left(\Delta_{0} / \eta=32\right)$ and $2000\left(\Delta_{0} / \eta=64\right)$
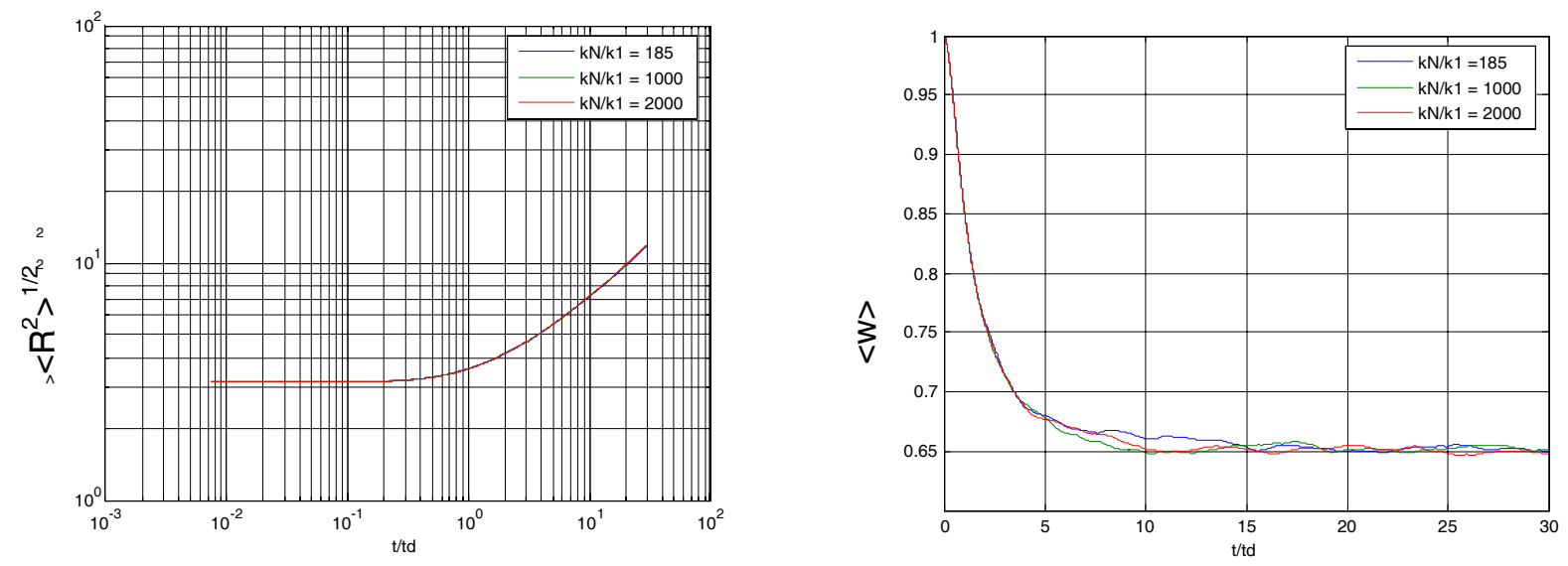

Fig.21. Time evolution of the triangle size and shape factor $\langle w\rangle$ for initial separation

$\Delta_{0} / \mathrm{L}_{1}=0.5, \mathrm{St}=0.02$ and drift velocity parameter $\mathrm{W}=2$ at different inertia subranges $k_{N} / k_{1}=185\left(\Delta_{0} / \eta=92.5\right), 1000\left(\Delta_{0} / \eta=500\right)$ and $2000\left(\Delta_{0} / \eta=1000\right)$

All curves for constant values of $\Delta_{0} / \mathrm{L}_{1}=0.032$ and $\Delta_{0} / \mathrm{L}_{1}=0.5$ are approximately collapse for the plotted values of $\left\langle R^{2}\right\rangle^{1 / 2},\langle W\rangle$ and $\langle\chi\rangle$ for the range of $0.2<W<4$ at $\mathrm{St}=0.02$. These results are obtained of for a three-particle cluster but in case of a cluster diffusion (Nicolleau and EIMaihy [5]) we extend these finding to the heavy particle dispersion for drift velocities in the range of $0.2<\mathrm{W}<4$ at a Stokes number $\mathrm{St}=0.02$ in the range of inertial range of $185<\mathrm{k}_{N} / \mathrm{k}_{1}<2000$.

\section{CONCLUSION:}

In the present work, the effect of particle inertia in the absence of gravity and the effect of particle drift velocity in the presence of particle inertia on the dispersion of three-particle clusters in a homogenous isotropic turbulent flow are studied. The evolution of the size and the shape of an initially equilateral triangle immersed in isotropic turbulence are presented.

For the effect of particle inertia in the absence of gravity, the dispersion of heavy particles in turbulent flow show that:

1. Inertia affects the size and shape of the three-particle cluster in turbulent flow. If the initial separation is above Kolmogorov length-scale, the size of the cluster in dispersion process is larger than diffusion process in the turbulent flow. If the initial separation is below Kolmogorov length-scale, there will be a critical time for the size and shape of the cluster a before which dispersion process is more important and after which the diffusion process which is more significant. This critical time decreases when the initial separation decreases,

2. At constant Stokes number, with the increase of initial separation, the size reaches the asymptotic values without experiencing the Richardson regime. The minimum values of the shape factors $\mathrm{w}, \mathrm{I}_{2}$ and $\chi$ decrease with the increase of initial separation, by continuing increase of initial separation 
these parameters reach their asymptotic value directly without passing through a minimum value,

3. We can extend the validity of the obtained values by Nicolleau and EIMaihy [5] $\left(0.66\right.$ for $\langle w\rangle, 0.16$ for $\left\langle\left.\right|_{2}\right\rangle$ and 0.26 for $\langle x\rangle$ ) to the dispersion of heavy particle clusters in the case of varying Stokes number at zero drift velocity,

4. At fixed initial separation, with the increase of inertia, the minimum value of shape factor $w$ is increases. Consequently, they tend to reach asymptotic value faster than in case of diffusion. For the effect of Reynolds number on the dispersion of heavy particle clusters, we extend Nicolleau and EIMaihy [5] findings [all curves for constant values of initial separations of $\Delta_{0} / L_{1}=0.032$ and $\Delta_{0} / L_{1}=0.5$ are approximately collapsed for factors $\left\langle R^{2}\right\rangle^{1 / 2},\langle w\rangle$ and $\langle\chi\rangle$ ] to the heavy particle dispersion of Stokes numbers in the range $0.2<\mathrm{St}<1$ at zero drift velocity, for the range of inertial ranges $185<\mathrm{k}_{N} / \mathrm{k}_{1}<2000$, but we limit the above conclusion to initial separations above the Kolmogorov lengthscale only.

For the particle drift velocity in the presence of particle inertia, the dispersion of heavy particles in turbulent flow shows that:

1. The drift velocity affects the size and shape of the three-particle cluster in turbulent flow, with the increase of drift velocity, whether the initial separation is below or above the Kolmogorov length scale, the dispersion process enhances the size distortion of the cluster by reaching the asymptotic value faster than the diffusion process and the cluster reaches the minimum value faster than the diffusion,

2. With the increase of initial separation the size reaches the largest length scale and is going towards the asymptotic values without experiencing the Richardson regime. Also for the shape factor at a constant drift velocity and Stokes number, it is noticed that the minimum value of factors $\mathrm{w}, \mathrm{I}_{2}$ and $\chi$ decreases with the increase of initial separation, by continuing the increase of initial separation these parameters reach the asymptotic value directly without passing through a minimum value,

3. All the curves for constant values of $\Delta_{0} / L_{1}=0.032$ and $\Delta_{0} / L_{1}=0.5$ are approximately collapse for the plotted value of $\left\langle R^{2}\right\rangle^{1 / 2},\langle W\rangle$ and $\langle\chi\rangle$ in the range of $0.2<\mathrm{W}<4$ at St $=0.02$ so we extend the finding in case of a cluster diffusion (Nicolleau and EIMaihy [5]) to the heavy particle dispersion for drift velocities in the range of $0.2<\mathrm{W}<4$ at a Stokes number $\mathrm{St}=0.02$ in the range of inertial range of $185<\mathrm{k}_{\mathrm{N}} / \mathrm{k}_{1}<2000$.

\section{REFERENCES:}

[1] Mydlarski, L., Pumir, A., Shraiman, B., Siggia, E.D. and Warhaft, Z., "Structure and Multipoint Correlators for Turbulent Advection: Prediction and Experiments." Physical Review Letters, 81(20), 4373-4376, (1998).

[2] Pumir, A., Shraiman, B.I. and Chertkov, M., "Geometry of Lagrangian Dispersion in Turbulence" Physical Review Letters, 85(25), 5324-5327, (2000).

[3] Castiglione, P. and Pumir A., "Evolution of Triangles in a Two-Dimensional Turbulent Flow." Physical Review E, 64, 056303, (2001).

[4] Khan, M.A., Pumir, A. and Vassilicos, J.C., "Kinematic Simulation of Turbulent Dispersion of Triangles." Physical Review E, 68, 026313, (2003). 
[5] Nicolleau, F. and ElMaihy, A., "Effect of the Reynolds Number on Three- and Four-Particle Diffusion in Three-Dimensional Turbulence Using Kinematic Simulation." Physical Review E, 74, 046302, (2006).

[6] Fung, J.C.H., Hunt, J.C.R., Malik, N.A. and Perkins, R.J., "Kinematic Simulation of Homogeneous Turbulence by Unsteady Random Fourier Modes." Journal of Fluid Mechanics, 236, 281-317, (1992).

[7] Kraichnan, R.H., "Diffusion by a Random Velocity Field." Physics of Fluids, 13, 22-31, (1970).

[8] Drummond, I., Duane, S. and Horgan, R., "Scalar Diffusion in Simulated Helical Turbulence with Molecular Diffusivity." Journal of Fluid Mechanics, 138, 75-91, (1984).

[9] Fung, J.C.H. and Vassilicos, J.C., "Two-Particle Dispersion in Turbulent-like Flows." Physical Review E, 57, 1677-1690, (1998).

[10] Malik, N.A. and Vassilicos, J.C., "A Lagrangian Model for Turbulent Dispersion with Turbulent-like Flow Structure: Comparison with DNS for Two Particle Statistics." Physics of Fluids, 11, 1572-1580, (1999).

[11] Flohr, P. and Vassilicos, J.C., "A Scalar Subgrid Model with Flow Structure for Large-Eddy Simulations of Scalar Variances." Journal of Fluid Mechanics, 407, 315-349, (2000).

[12] Fung, J.C.H., "Kinematic Simulation of Turbulent Flow and Particle Motion." Ph.D. dissertation, University of Cambridge, Cambridge, UK, (1990).

[13] Maxey, M.R. and Riley, J.J., "Equation of Motion for a Small Rigid Sphere in a Nonuniform Flow." Physics of fluid, 26, 883-889, (1983).

[14] Maxey, M.R., "The Equation of Motion for a Small Rigid Sphere in a Nonuniform or Unsteady Flow" ASME/FED, Gas-Solid Flows, 166, 57-62, (1993).

[15] EIMaihy, A., " Study of Diffusion and Dispersion of Particles Using Kinematic Simulation." Ph.D. dissertation, The University of Sheffield, Sheffield, UK, (2003).

[16] Pumir, A., "Structure of Three-Point Correlation Function of a Passive Scalar in the Presence of Mean Gradient." Physical Review E, 57(3), 2914-2929, (1998).

[17] Yudine, M. I. (1959). "Physical Consideration on Heavy-particle Dispersion." Advances in Geophysics, 6, 185-191. 